SPhT 97/078

\title{
Generalized Dynkin diagrams and root systems and their folding
}

\author{
Jean-Bernard Zuber \\ CEA Saclay, Service de Physique Théorique \\ F-91191 Gif sur Yvette Cedex, France
}

\begin{abstract}
Graphs which generalize the simple or affine Dynkin diagrams are introduced. Each diagram defines a bilinear form on a root system and thus a reflection group. We present some properties of these groups and of their natural "Coxeter element". The folding of these graphs and groups is also discussed, using the theory of C-algebras.
\end{abstract}

Submitted for publication in the proceedings of the Taniguchi Symposium Topological Field Theory, Primitive Forms and Related Topics, Kyoto Dec 1996, M. Kashiwara, A. Matsuo, K. Saito and I. Satake eds, Birkhaüser. 


\title{
Generalized Dynkin diagrams and root systems and their folding
}

\author{
Jean-Bernard Zuber
}

\begin{abstract}
Graphs which generalize the simple or affine Dynkin diagrams are introduced. Each diagram defines a bilinear form on a root system and thus a reflection group. We present some properties of these groups and of their natural "Coxeter element". The folding of these graphs and groups is also discussed, using the theory of C-algebras.
\end{abstract}

\section{Introduction}

This paper is devoted to the study of generalized Dynkin diagrams, regarded as encoding the geometry of a root system, and of the ensuing reflection groups. The graphs are $N$-colourable -in a sense to be defined below- while the ordinary simple or affine Dynkin diagrams correspond to the case $N=2$. In fact, the main features of the construction are based on the algebra $\operatorname{sl}(N)$ or its affine extension $\widehat{s l}(N)$, with the ordinary (simple or affine) Dynkin diagrams and finite or affine reflection groups being associated with $\operatorname{sl}(2)$. Ordinary $A D E$ (resp affine $\hat{A} \hat{D} \hat{E}$ ) Dynkin diagrams are known to be the only bi-colourable graphs with a spectrum of their adjacency matrix less than (resp. less than or equal to) 2. Here we use an appropriate generalization of this spectral property.

This generalization has emerged in a natural way in several related problems of current interest in mathematical physics: conformal field theories, integrable lattice models, topological field theories (for a review, see for example [Zi]) and 3-manifold invariants [O].

In section 1 we introduce the notations and the axioms imposed on the graphs. Section 2 presents the main results on the associated reflection groups: signature of the metric, definition of a natural Coxeter element and its order. In section 3 is discussed the folding of ordinary and generalized Dynkin diagrams. While the folding of simply laced into non simply laced Dynkin diagrams, for example $E_{8}$ into $H_{4}$, is usually found in some empirical way $[\mathrm{Y}, \mathrm{Sh}, \mathrm{MP}]$, it is shown that there is a general way to find such possible foldings, based on the structure of the so-called "C-algebras" [BI] attached to the graph (for a review, see 
for example $[\mathrm{Zr}])$. Some aspects of the connection with singularities are discussed in sect. 4. as well as some indications about the connections with superconformal and topological field theories.

This paper is an extension of a former exposition [Z] but may be read independently. The main new results concern: i) the extension of the discussion from graphs generalizing the ordinary Dynkin diagrams of finite type to a larger class encompassing the graphs of affine type; ii) the discussion of the folding of (generalized) Dynkin diagrams and reflection groups.

\section{Generalized Dynkin diagrams}

\subsection{Notations on $\operatorname{sl}(N)$}

We shall consider a class of graphs which generalize the classical $A, D, E$ and extended $\hat{A}, \hat{D}, \hat{E}$ Dynkin diagrams. While the $A D E$ or $\hat{A} \hat{D} \hat{E}$ diagrams may be regarded as related to the $s l(2)$ algebra, the new ones are associated with $\operatorname{sl}(N)$. Let $\Lambda_{1}, \cdots, \Lambda_{N-1}$ be the fundamental weights of $\operatorname{sl}(N)$. Let $\rho=\Lambda_{1}+\cdots+\Lambda_{N-1}$ denote their sum. I denote the weight lattice by $\mathcal{P}$. Let $k \in \mathbb{N}$. I recall that the set of integrable weights (shifted by $\rho$ ) of the affine algebra $\widehat{s l}(N)$ at level $k$ is the following subset of $\mathcal{P}$

$\mathcal{P}_{++}^{(h)}=\left\{\lambda=\lambda_{1} \Lambda_{1}+\cdots+\lambda_{N-1} \Lambda_{N-1} \mid \lambda_{i} \in \mathbb{N}, \lambda_{i} \geq 1, \lambda_{1}+\cdots+\lambda_{N-1} \leq h-1\right\}$,

where $h=k+N$. We shall also consider the larger set

$$
\mathcal{P}_{+}^{(h)}=\left\{\lambda=\lambda_{1} \Lambda_{1}+\cdots+\lambda_{N-1} \Lambda_{N-1} \mid \lambda_{i} \in \mathbb{N}, \lambda_{i} \geq 0, \lambda_{1}+\cdots+\lambda_{N-1} \leq h\right\} .
$$

These two sets admit two natural automorphisms:

the conjugation $\mathcal{C}$ of representations

$$
\mathcal{C}: \quad \lambda=\left(\lambda_{1}, \lambda_{2}, \cdots, \lambda_{N-1}\right) \mapsto \bar{\lambda}=\left(\lambda_{N-1}, \cdots, \lambda_{2}, \lambda_{1}\right)
$$

and the $\mathbb{Z}_{N}$ automorphism

$$
\sigma: \quad \lambda=\left(\lambda_{1}, \lambda_{2}, \cdots, \lambda_{N-1}\right) \mapsto \lambda^{\prime}=\left(h-\sum_{j=1}^{N-1} \lambda_{j}, \lambda_{1}, \lambda_{2}, \cdots, \lambda_{N-2}\right) .
$$

We then introduce the weights $e_{i}$ of the standard $N$-dimensional representation of $\operatorname{sl}(N)$

$$
e_{1}=\Lambda_{1}, \quad e_{i}=\Lambda_{i}-\Lambda_{i-1},(i=2, \cdots, N-1), \quad e_{N}=-\Lambda_{N-1}
$$

endowed with the scalar product $\left(e_{i}, e_{j}\right)=\delta_{i j}-\frac{1}{N}$. 


\subsection{Axioms on the graphs}

The graphs are defined by the following data and axioms

1) A set $\mathcal{V}$ of $|\mathcal{V}|=n$ vertices is given. These vertices are denoted by latin letters $a, b, \cdots$ There exists an involution $a \mapsto \bar{a}$ and the set $\mathcal{V}$ admits a $\mathbb{Z}_{N}$ grading denoted $\tau(a)$ and called $N$-ality or color, such that $\tau(\bar{a})=-\tau(a) \bmod N$.

2) A set of $N-1$ commuting $n \times n$ matrices $G_{p}$ is given. They may be thought of as labelled by the fundamental representations of $\operatorname{sl}(N)$, $G_{p}=G_{\square}, 1 \leq p \leq N-1$. Their matrix elements are assumed to be $::\} p$

non negative integers, so that they may be regarded as adjacency matrices of $N-1$ graphs $\mathcal{G}_{p}$. Multiple edges $\left(G_{p}\right)_{a b} \geq 2$ are allowed. (The integrality assumption may be relaxed to allow the analogue of non simply-laced Coxeter-Dynkin diagrams, see below Remark 2.) We also assume that the graph $\mathcal{G}_{1}$ is connected.

3) The edges of the graphs $\mathcal{G}_{p}$ are compatible with the grading $\tau$ in the sense that

$$
\left(G_{p}\right)_{a b}=0 \quad \text { if } \quad \tau(b) \neq \tau(a)+p \bmod N .
$$

Thus for $p \neq \frac{N}{2}$, the edges are oriented. Also note that for a given pair $(a, b)$, there is at most one graph $\mathcal{G}_{p}$ with an edge between $a$ and $b$.

4) The matrices are transposed of one another

$$
G_{p}^{t}=G_{N-p}
$$

and are invariant under the involution $a \mapsto \bar{a}$ in the sense that

$$
\left(G_{p}\right)_{\bar{a} \bar{b}}=\left(G_{p}\right)_{b a}
$$

5) As a consequence of 2) and 4), the matrices $G_{p}$ commute with their transpose ("normal matrices") and may thus be simultaneously diagonalized in a common orthonormal basis. This basis, denoted $\psi^{(\lambda)}$, is assumed to be labelled by weights $\lambda$ of $\operatorname{sl}(N)$, that are restricted to be in $\mathcal{P}_{+}^{(h)}$, -or even more restricted to be in $\mathcal{P}_{++}^{(h)}$ (see below)-, for some integer $h \geq N$, in such a way that the eigenvalues $\gamma_{p}^{(\lambda)}$ have the form

$$
\gamma_{p}^{(\lambda)}=\chi_{p}(M(\lambda))
$$

where $\chi_{p}$ is the ordinary character for the $p$-th fundamental representation of the group $S U(N)$, and $M(\lambda)$ denotes the diagonal matrix 
$M(\lambda)=\operatorname{diag}\left(\varepsilon_{j}(\lambda)\right)_{j=1, \cdots N}$. Here and throughout this article,

$$
\varepsilon_{j}(\lambda):=\exp -\frac{2 i \pi}{h}\left(e_{j}, \lambda\right)
$$

Maybe more explicitly, the $\gamma_{p}$ read

$$
\begin{array}{rlr}
\gamma_{1}^{(\lambda)}=\sum_{i=1}^{N} \exp -\frac{2 i \pi}{h}\left(e_{i}, \lambda\right) & =\chi_{1}(M) \\
\gamma_{2}^{(\lambda)}=\sum_{1 \leq i<j \leq N} \exp -\frac{2 i \pi}{h}\left(\left(e_{i}+e_{j}\right), \lambda\right) & =\chi_{2}(M) \\
\vdots & \vdots \quad \vdots \\
\gamma_{N-1}^{(\lambda)}=\sum_{1 \leq i_{1}<\cdots i_{N-1} \leq N} \exp -\frac{2 i \pi}{h}\left(\left(e_{i_{1}}+\cdots+e_{i_{N-1}}\right), \lambda\right) \\
=\left(\gamma_{1}^{(\lambda)}\right)^{*} & =\chi_{N-1}(M) .
\end{array}
$$

We call $h$ the "Coxeter number" of the graph. Also we call these weights $\lambda$ "exponents", and denote their set Exp. Some of these exponents may occur with multiplicities. The class of graphs for which all the exponents belong to $\mathcal{P}_{++}^{(h)}$ is referred to as class II, those for which they belong to $\mathcal{P}_{+}^{(h)}$ (with some belonging to $\mathcal{P}_{+}^{(h)} \backslash \mathcal{P}_{++}^{(h)}$ ) form the class I.

6) We assume that the weight 0 for the class I, or $\rho$ for the class II, is an exponent, with multiplicity 1 . The corresponding eigenvalue reads

$$
\begin{array}{rlrl}
\gamma_{p}^{(0)} & =\left(\begin{array}{l}
N \\
p
\end{array}\right) & \text { for class I } \\
\text { and } \quad \gamma_{p}^{(\rho)} & =\left(\begin{array}{l}
N \\
p
\end{array}\right)_{q} \quad \text { for class II }
\end{array}
$$

in terms of a $q$-deformed binomial coefficient $q=e^{\frac{i \pi}{h}}$,

$$
\left(\begin{array}{c}
N \\
p
\end{array}\right)_{q}=\frac{\sin \frac{\pi N}{h} \cdots \sin \frac{\pi(N-p+1)}{h}}{\sin \frac{\pi}{h} \sin \frac{2 \pi}{h} \cdots \sin \frac{p \pi}{h}} .
$$

Remark 1. By an abuse of language, what is called "a graph" in the previous description is in fact the collection of $N-1$ graphs, with adjacency matrices $\mathcal{G}_{p}$, connecting the given set of vertices $\mathcal{V}$. Note that 
if the grading $\tau$ of each vertex is known, one may simply consider the unique graph of adjacency matrix

$$
G=G_{1}+\cdots+G_{N-1}
$$

Each matrix $G_{p}$ is then identified as made of the matrix elements $G_{a b}$ of $G$ that satisfy $\tau(b)-\tau(a)=p \bmod N$.

Remark 2. As mentioned in 2), it is natural to relax the assumption of integrality of the matrix elements $\left(G_{p}\right)_{a b}$ and to allow values of the

form either $2 \cos \frac{\pi}{m_{a b}}\left(m_{a b} \in \mathbb{N}\right)$ or $\left(\begin{array}{l}N \\ p\end{array}\right)_{q}$ (see (13) and sect. 3.4 below). By a small abuse of language, we still call $G$ the adjacency matrix of a graph, whose edges are decorated by some marking, e.g. $\bullet m_{a b} \circ$ in the first case. This is a generalization of the distinction between simply and non simply laced Coxeter-Dynkin diagrams. Note that now the name "(non) simply laced" is inappropriate and should be replaced by "(non) integrally laced", as there are graphs whose adjacency matrix may have entries equal to 2 (see examples below).

\subsection{Examples}

1. Examples of class I for $N=2$ are provided by the affine Dynkin diagrams, $\hat{A}_{2 p-1}, \hat{D}_{n}, \hat{E}_{6}, \hat{E}_{7}, \hat{E}_{8}$. (The restriction to odd $2 p-1$ in $\hat{A}$ comes from the assumption that the graph is bicolorable ( $\mathbb{Z}_{2}$ grading)). The conjugation $a \rightarrow \bar{a}$ is trivial in these cases: $\bar{a} \equiv a$. If the assumption of integrality of the entries of the $G_{p}$ matrices is relaxed, one finds also the $\hat{B}_{n}, \hat{C}_{n}, \hat{F}_{4}$ and $\hat{G}_{2}$ Dynkin diagrams. The $\hat{A}, \hat{D}$ and $\hat{E}$ cases are known to be in one-to-one correspondence with the finite subgroups of $S U(2)[\mathrm{MK}]$.

The Coxeter number and exponents of the $\hat{A}, \hat{D}$ and $\hat{E}$ cases are as follows

$\begin{array}{lll}\hat{A}_{2 n-1} & n & 0,1, \cdots n-1, n, n-1, \cdots 1 . \\ \hat{D}_{n+2} & 2 n & 0,2, \cdots 2 n ; n, n \\ \hat{E}_{6} & 6 & 0,2,2,3,4,4,6 \\ \hat{E}_{7} & 12 & 0,3,4,6,6,8,9,12 \\ \hat{E}_{8} & 30 & 0,6,10,12,15,18,20,24,30\end{array}$

More generally, any finite subgroup $H$ of $S U(N)$ containing its center $\mathbb{Z}_{N}$ yields a solution of class I. The set $\mathcal{V}=\left\{R_{a} ; a=1, \cdots, n\right\}$ is the set of irreducible representations of $H$. As $H$ is embedded into $S U(N)$, one also knows $N-1$ special representations $\mathcal{R}_{p}$ of $H$, obtained by restriction of the $N-1$ fundamental representations of $S U(N)$ to $H$. Note that these representations are not necessarily irreducible. The matrix $G_{p}$ is then defined as providing the decomposition into irreducible representations 
of the tensor product by $\mathcal{R}_{p}$

$$
\mathcal{R}_{p} \otimes R_{a}=\oplus_{b}\left(G_{p}\right)_{a b} R_{b}
$$

The axioms 1)-6) are satisfied. The involution $a \mapsto \bar{a}$ is the conjugation of representations. The grading $\tau(a)$ is the " $N$-ality" of the representation $R_{a}$ defined by

$$
\text { if } \xi \in \mathbb{Z}_{N} \subset H \quad R_{a}(\xi)=\xi^{\tau(a)} \mathbb{I}
$$

and satisfies $\tau(\bar{a})=-\tau(a) \bmod N$. The matrices commute, as a consequence of the commutativity of the tensor product. The graph $\mathcal{G}_{1}$ is connected: as the $N$-dimensional fundamental representation is faithful, all representations of $H$ appear in the decomposition of its repeated tensor powers $[\mathrm{FH}]$. Properties 3) and 4) are simple consequences of the fact that $\left(G_{p}\right)_{a b}$ is the multiplicity of the identity representation in the product $\mathcal{R}_{p} \otimes R_{a} \otimes R_{\bar{b}}$. The eigenvalues of the matrices $G_{p}$ are the fundamental characters of $S U(N), \chi_{p}\left(M_{\alpha}\right)$, evaluated for a representative of the class $\alpha$ of $H$. As $\chi_{p}$ is a class function in $S U(N)$, one may compute $\chi_{p}\left(M_{\alpha}\right)$ in terms of the eigenvalues of $M_{\alpha} \sim \operatorname{diag}\left(\exp \epsilon_{j}\right)_{j=1, \cdots, N}$, $\sum_{j} \epsilon_{j}=0$. These eigenvalues have the form $\epsilon_{j}=-\frac{2 i \pi n_{j}}{|H|}, n_{j} \in \mathbb{Z}$, since any element of $H$ is at most of order $|H|: M_{\alpha}^{|H|}=\mathbb{I}$; the $n$ 's are defined modulo $|H|$ and up to a permutation. One may identify

$$
\begin{aligned}
\frac{n_{i}}{|H|} & =\frac{\left(e_{i}, \lambda\right)}{h} \quad \text { for some } \lambda \in \mathcal{P} \text { and some } h \in \mathbb{N} \\
& =\frac{1}{h N}\left(\sum_{j=i}^{N-1} \lambda_{j}(N-j)-\sum_{j=1}^{i-1} j \lambda_{j}\right)
\end{aligned}
$$

by taking $\lambda_{i}=h \frac{\left(n_{i}-n_{i+1}\right)}{|H|}$. $h$ is chosen to be the smallest common denominator of the fractions $\left(n_{i}-n_{i+1}\right) /|H|$, i.e. $h$ always divides $|H|$. The resulting $\lambda$ is not necessarily in $\mathcal{P}_{+}^{(h)}$. One may however find an image $\mu$ of $\lambda$ by the affine Weyl group of $s l(N)$ such that $\mu \in \mathcal{P}_{+}^{(h)}$ and $\gamma_{p}^{(\lambda)}=\gamma_{p}^{(\mu)}$ (see below the proof of lemma 1.1). This completes the proof that axiom 5 is indeed satisfied. Finally any subgroup of $S U(N)$ contains the class of the identity for which $\lambda=0$, with multiplicity 1 .

As an example of a case with $N>2$, the graph associated with the subgroup $\Sigma(1080)$ of $S U(3)$ (with the notations of $[\mathrm{FFK}]$ ) is exhibited in Fig.1(a). Its Coxeter number is $h=60$ and its $n=17$ exponents are $(0,0),(15,15),(30,30),(6,6),(12,12)$ and their transforms by $\sigma$, and $(10,10)$ with multiplicity 2 . Drawings of more graphs pertaining to $S U(3)$ may be found in [DFZ1]. 


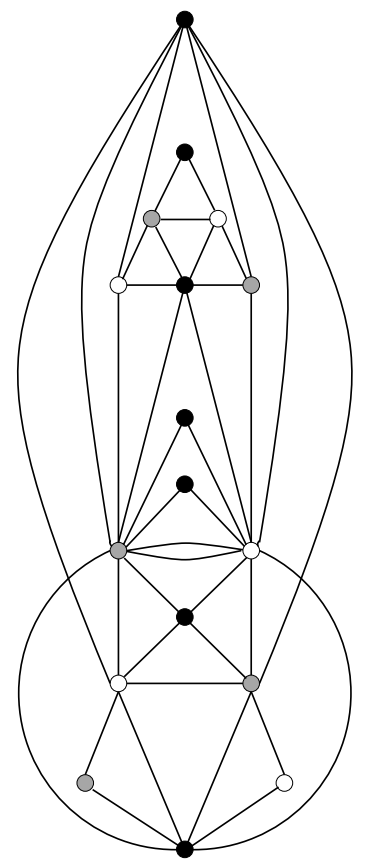

(a)

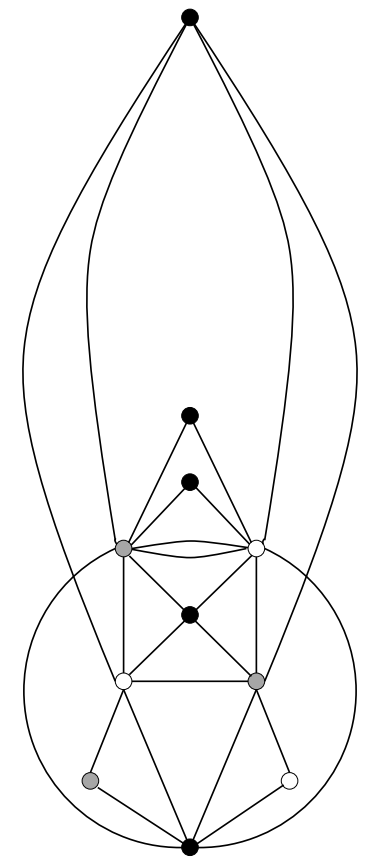

(b)

Fig. 1: The graph associated with the subgroup $\Sigma(1080)$ (a) and its truncated counterpart of class II (b). The three colours -black, grey and white- mark vertices with $\tau=0,1,2$ respectively. The exponents of graph (b) are $(1,1),(3,3),(5,5)$ and their $\sigma$-orbit and twice $(4,4)$.

2. Examples of class II for $N=2$ are provided by the ordinary simply laced Dynkin diagrams, $A_{n}, D_{n}, E_{6}, E_{7}, E_{8}$. If the assumption of integrality of the entries of the $G_{p}$ matrices is relaxed, one finds also the $B_{n}$, $C_{n}, F_{4}, G_{2}, H_{3}, H_{4}$ and $I_{2}(h)$ Coxeter-Dynkin diagrams, with the identifications $B_{n}=C_{n}$ (between their symmetrized Cartan matrices), and $G_{2}=I_{2}(6)$. This in fact exhausts all the (bicolorable) graphs satisfying the axioms for $N=2[\mathrm{GHJ}]$.

There exists another class of solutions known for all $N$, namely the fusion graphs of the affine algebra $\widehat{s l}(N)$ at level $k$. The vertices are the integrable weights described above, i.e. $\mathcal{V}=\mathcal{P}_{++}^{(h)}, h=k+N$. The definitions of the involution and of the grading are the same as in Example 1: the involution is $\lambda \mapsto \bar{\lambda}$ defined in (3) and the grading may be chosen as $\tau(\lambda)=\sum_{j=1}^{N-1} j\left(\lambda_{j}-1\right) \bmod N$. The matrices $G_{p}$ are the Verlinde matrices, which describe the fusion, rather than the ordinary tensor product, by the $p$-th fundamental representation. Their diagonalization is known, thanks to the Verlinde formula [Ve], to be achieved by the unitary matrix $S_{\lambda}{ }^{\mu}$ of modular transformations of affine characters $[\mathrm{KP}]$, and the eigenvalues are the $\gamma_{p}^{(\lambda)}$, where $\lambda$ takes itself all the 
values in $\mathcal{P}_{++}^{(h)}$. (This case enjoys self-duality properties, in agreement with the fact that both the vertex set $\mathcal{V}$ and the set of exponents Exp are isomorphic to $\mathcal{P}_{++}^{(h)}$.) In the case $N=2$, these fusion graphs reduce to the $A_{h-1}$ Dynkin diagram.
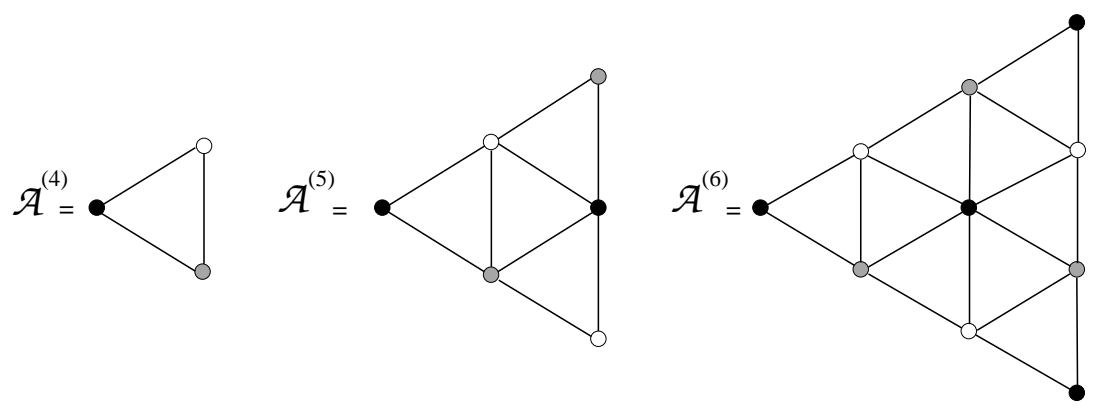

Fig. 2: The first fusion graphs of $\widehat{s l}(3)$. Same convention as in Fig. 1
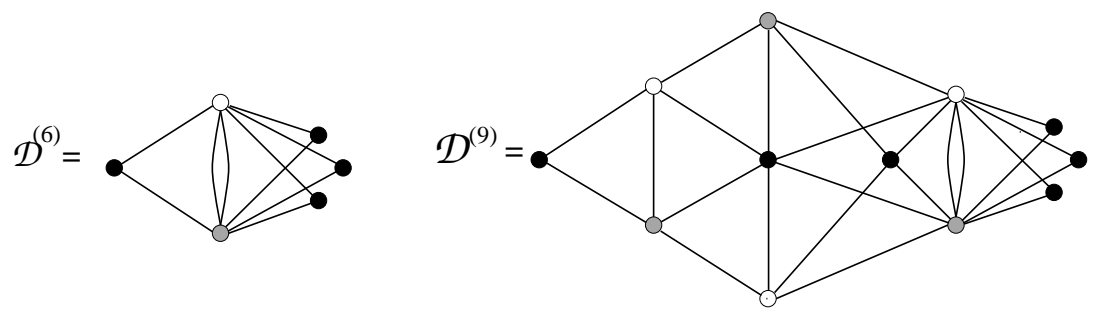

Fig. 3: The first orbifolds of $\widehat{s l}(3) . \mathcal{D}^{(6)}$ is the $\mathbb{Z}_{3}$ orbifold of the graph $\mathcal{A}^{(6)}$ of Fig. 2.

More solutions are known, which generalize the two previous cases of $A D E$ Dynkin diagrams and of fusion graphs. Infinite series - which are the analogues of the $D$ Dynkin diagrams- have been obtained by orbifolding the fusion graphs [Ko], see Fig $3^{1}$. Several others have been obtained, originally by empirical methods [DFZ1], starting from graphs of finite subgroups of $S U(N)$ (cf. Example 1) and removing some vertices and edges in order to attain the spectral properties of axioms 5-6. For example, a graph related to the previously subgroup $\Sigma(1080)$ is presented in Fig 1(b). It has $h=12$. Recently, more systematic methods have been set up, which make use of some data of rational conformal theory to construct graphs [PZ]. There is, however, a shortage of general results on the class of graphs satisfying the previous axioms.

\footnotetext{
1 It is amusing to notice that the graph $\mathcal{D}^{(6)}$ has already been encountered in another systematic study of "extended affine root systems" [S]: this is the graph denoted there $D_{4}^{(1,1)}$.
} 


\section{Problems:}

1. Classify all the solutions to the axioms 1-6.

This is presumably too difficult. More modest questions are:

2. Are all solutions of class I obtained from some finite subgroup of $S U(N)$ ?

3. Are all solutions of class II obtained by truncation of some solution of class I?

It appears that the answers to 2 and 3 cannot be both "yes". In [DFZ1], solutions of class II were found for $N=3$ which do not seem to emanate from a known subgroup of $S U(3)$.

\subsection{Simple consequences of the axioms}

Lemma 1.1 : The set of $\gamma_{p}^{(\lambda)}$, for all $p=1, \cdots, N-1$, characterizes the weight $\lambda$ in $\mathcal{P}_{+}^{(h)}$ :

$$
\text { if for all } \quad p=1, \cdots, N-1 \quad \gamma_{p}^{(\lambda)}=\gamma_{p}^{(\mu)}, \text { then } \quad \lambda=\mu \text {. }
$$

Proof: The $\gamma_{p}^{(\lambda)}, p=1, \cdots, N$ (with $\gamma_{N}^{(\lambda)}=1$ ), are the elementary symmetric functions of the $\varepsilon_{i}(\lambda)=\exp -\frac{2 i \pi}{h}\left(e_{i}, \lambda\right), i=1, \cdots, N$. Thus $\gamma_{p}^{(\lambda)}=\gamma_{p}^{(\mu)}$ for all $p=1, \cdots, N$ implies that $\varepsilon_{i}(\lambda)$ is a permutation of the $\varepsilon_{i}(\mu)$. Since the Weyl group of $s l(N)$ acts on the $e_{i}$ by permutation, this means that there is an element $w$ of the (finite) Weyl group such that

$$
\varepsilon_{i}(\mu)=e^{-\frac{2 i \pi}{h}\left(e_{i}, \mu\right)}=e^{-\frac{2 i \pi}{h}\left(w e_{i}, \lambda\right)}=e^{-\frac{2 i \pi}{h}\left(e_{i}, w^{-1} \lambda\right)},
$$

i.e. for all $i,\left(e_{i}, \mu\right)=\left(e_{i}, w^{-1} \lambda\right)+n_{i} h,\left(n_{i} \in \mathbb{Z}, \sum n_{i}=0\right)$. Thus there is an element $\tilde{w}$ of the affine Weyl group such that $\mu=\tilde{w} \lambda$. Since $\mathcal{P}_{+}^{(h)}$ is a fundamental domain of the weight lattice under the action of the affine Weyl group, we conclude that if both $\lambda$ and $\mu$ belong to that domain, they are identical. q.e.d. By the same token, we learn that any weight $\lambda \in \mathcal{P}$ has an image $\mu$ by the affine Weyl group that belongs to $\mathcal{P}_{+}^{(h)}$ such that $\gamma_{p}^{(\lambda)}=\gamma_{p}^{(\mu)}$ for all $p$.

Now consider the set Exp. One has the following

Proposition 1.2: The set Exp is invariant under the action of $\sigma$ and of $\mathcal{C}$. In other words, the set Exp contains full orbits of $\sigma$ and of $\mathcal{C}$. Moreover the eigenvectors may be chosen so as to satisfy

$$
\begin{gathered}
\psi_{a}^{(\bar{\lambda})}=\psi_{\bar{a}}^{(\lambda)}=\left(\psi_{a}^{(\lambda)}\right)^{*} \\
\psi_{a}^{(\sigma(\lambda))}=e^{\frac{2 i \pi}{N} \tau(a)} \psi_{a}^{(\lambda)} .
\end{gathered}
$$

This follows from the fact that $\left(e_{i}, \sigma(\lambda)\right)=\left(e_{i-1}, \lambda\right)-\frac{h}{N}$ for $i=2, \cdots, N$ 
and $\left(e_{1}, \sigma(\lambda)\right)=\left(e_{N}, \lambda\right)+\frac{N-1}{N} h$, whence

$$
\begin{aligned}
\varepsilon_{i}(\sigma(\lambda)) & =e^{\frac{2 i \pi}{N}} \varepsilon_{i-1}(\lambda) \\
\varepsilon_{i}(\lambda)^{*} & =\varepsilon_{N+1-i}(\bar{\lambda})
\end{aligned}
$$

and thus

$$
\begin{aligned}
\gamma_{p}^{(\sigma(\lambda))} & =e^{2 i \pi \frac{p}{N}} \gamma_{p}^{(\lambda)} \\
\text { and } \gamma_{p}^{(\bar{\lambda})} & =\left(\gamma_{p}^{(\lambda)}\right)^{*} ;
\end{aligned}
$$

then from axiom 3), if $\psi_{a}^{(\lambda)}$ is an eigenvector for the eigenvalue $\gamma_{p}^{(\lambda)}$ of $G_{p}$, all $p$, so is $\tilde{\psi}_{a}^{(\lambda)}:=e^{2 i \pi \frac{\tau(a)}{N}} \psi_{a}^{(\lambda)}$ for the eigenvalue $\gamma_{p}^{(\sigma(\lambda))}$. By lemma 1.1, this suffices to qualify $\sigma(\lambda)$ as an exponent. Likewise, the reality of the characteristic polynomials implies that $\gamma_{p}^{(\lambda)}$ and $\gamma_{p}^{(\lambda) *}$ are simultaneously eigenvalues, and thus $\lambda$ and $\bar{\lambda}$ are simultaneously exponents.

Corollary 1.3: The sum of exponents is

$$
\sum_{\operatorname{Exp}} \lambda=\frac{h n}{N} \rho
$$

where we recall that $n$ is the number of vertices and $\rho$ is the sum of all the fundamental weights.

Proof: Compute $\left(1+\sigma+\cdots+\sigma^{N-1}\right) \sum \lambda$ in two different ways. On the one hand, as $\operatorname{Exp}$ is invariant under $\sigma$, the sum equals $N \sum_{\text {Exp }} \lambda$. On the other, for each $\lambda,\left(1+\sigma+\cdots+\sigma^{N-1}\right) \lambda=h \rho$, thus the previous sum also equals $h n \rho$.

Corollary of corollary 1.3: $N$ divides $h n$.

For later use, I introduce an explicit parametrization of the matrices $G_{p}$. I assume that the vertices of $\mathcal{V}$ have been ordered according to increasing $\tau$ : first the vertices with $\tau=0$, then $\tau=1$, etc. Then the matrices $G_{p}$ are $N \times N$ block-matrices of the form

$$
G_{p}=\left(\begin{array}{cccccc}
0 & \cdots & 0 & A_{1 p+1} & \cdots & 0 \\
\vdots & \ddots & & & \ddots & \vdots \\
0 & \cdots & 0 & \cdots & 0 & A_{N-p N} \\
A_{N-p+11} & 0 & & 0 & \cdots & 0 \\
\vdots & \ddots & & 0 & \ddots & \vdots \\
0 & \cdots & A_{N p} & 0 & & 0
\end{array}\right)
$$

with the matrices $A_{i j}$ satisfying $A_{i j}^{t}=A_{j i}$ as a consequence of (7). (The matrices $A_{i j}$ are of course subject to further constraints expressing the 
commutation of the matrices $G$, etc). Later, we shall also encounter the matrix $^{2}$

$$
T=\left(\begin{array}{ccccc}
\mathbf{1} & A_{12} & A_{13} & \cdots & A_{1 N} \\
0 & \mathbf{1} & A_{23} & \cdots & A_{2 N} \\
& 0 & \ddots & & \\
& & 0 & \mathbf{1} & A_{N-1 N} \\
0 & 0 & \cdots & 0 & \mathbf{1}
\end{array}\right)
$$

It may be written as a product of upper triangular matrices in the two following ways:

$$
\begin{aligned}
T & =\left(\begin{array}{ccccc}
\mathbf{1} & & & & \\
& \mathbf{1} & & 0 & \\
& & \ddots & & \\
& 0 & & \mathbf{1} & A_{N-1 N} \\
& & & & \mathbf{1}
\end{array}\right) \cdots\left(\begin{array}{ccccc}
\mathbf{1} & A_{12} & A_{13} & \cdots & A_{1 N} \\
& \mathbf{1} & 0 & & 0 \\
& & \ddots & 0 & \\
& 0 & & \mathbf{1} & 0 \\
& & & & \mathbf{1}
\end{array}\right) \\
& =\left(\begin{array}{cccccc}
\mathbf{1} & & & & A_{1 N} \\
& \mathbf{1} & & 0 & A_{2 N} \\
& & \ddots & & \vdots \\
& 0 & & \mathbf{1} & A_{N-1 N} \\
& & & & \mathbf{1}
\end{array}\right) \cdots\left(\begin{array}{ccccc}
\mathbf{1} & A_{12} & 0 & \cdots & 0 \\
& \mathbf{1} & & 0 & \\
& & \ddots & & \\
& 0 & & \mathbf{1} & \\
& & & & \mathbf{1}
\end{array}\right),
\end{aligned}
$$

which allows to write its inverse and its transpose as

$$
T^{-1}=\left(\begin{array}{ccccc}
\mathbf{1} & -A_{12} & -A_{13} & \cdots & -A_{1 N} \\
& \mathbf{1} & 0 & & 0 \\
& & \ddots & 0 & \\
& 0 & & \mathbf{1} & \\
& & & & \mathbf{1}
\end{array}\right) \cdots\left(\begin{array}{ccccc}
\mathbf{1} & & & & \\
& \mathbf{1} & & 0 & \\
& & \ddots & & \\
& 0 & & \mathbf{1} & -A_{N-1 N} \\
& & & & \mathbf{1}
\end{array}\right)
$$

and

$$
T^{t}=\left(\begin{array}{ccccc}
\mathbf{1} & & 0 & \cdots & 0 \\
A_{21} & \mathbf{1} & & 0 & \\
& & \ddots & & \\
& 0 & & \mathbf{1} & \\
& & & & \mathbf{1}
\end{array}\right) \cdots\left(\begin{array}{ccccc}
\mathbf{1} & & & & \\
& \mathbf{1} & & 0 & \\
& & \ddots & & \\
& 0 & & \mathbf{1} & \\
A_{N 1} & A_{N 2} & \cdots & A_{N N-1} & \mathbf{1}
\end{array}\right) .
$$

\footnotetext{
2 Here and in the following, by a small abuse of notations, $\mathbf{1}$ denotes a unit matrix, whose dimension is fixed by the context.
} 


\section{Reflection Groups and Root Systems}

\subsection{Roots, bilinear form and reflection group.}

Given a graph of the previous type, we associate with it a vector space $V$ over $\mathbb{R}$, with a basis $\left\{\alpha_{a}\right\}$ labelled by the vertices $a$ of the set $\mathcal{V}$. A bilinear form is defined on the vectors of that basis by

$$
g_{a b}=\left\langle\alpha_{a}, \alpha_{b}\right\rangle=2 \delta_{a b}+G_{a b}
$$

in terms of the matrix $G=\sum_{p=1}^{N-1} G_{p} . \quad 3$ In terms of the matrix $T$ introduced in (25), one has simply

$$
g=T+T^{t}
$$

The vectors $\alpha_{a}$ will be called roots. For each root $\alpha_{a}$, one considers the reflection in the hyperplane through the origin orthogonal to $\alpha_{a}$

$$
S_{a}: x \in V \mapsto x^{\prime}=S_{a} x=x-\left\langle\alpha_{a}, x\right\rangle \alpha_{a}
$$

In components, if $x=\sum_{c} x_{c} \alpha_{c}$, then

$$
\begin{aligned}
& x_{a}^{\prime}=-x_{a}-\sum_{c \neq a} g_{a c} x_{c}=-x_{a}-\sum_{c \neq a} G_{a c} x_{c} \\
& x_{b}^{\prime}=x_{b} \quad \text { if } \quad b \neq a .
\end{aligned}
$$

One then considers the group $\Gamma$ generated by the $S_{a}, a=1, \cdots, n$.

Note that $S_{a}^{2}=\mathbb{I}$; if $G_{a b}=1$, then $\left(S_{a} S_{b}\right)^{3}=\mathbb{I}$ and more generally if $G_{a b}=2 \cos \frac{\pi p}{m}$, then $\left(S_{a} S_{b}\right)^{m}=\mathbb{I}$. The generators $S_{a}$ of the group may however satisfy additional relations [Z]. This would make the denomination "Coxeter group" improper, although as we shall see such groups share several properties with Coxeter groups.

3 Note that the sign differs from that used in [Z] if $N$ is even, and in particular for $N=2$, from that commonly used for the finite reflection groups for which $g$, the Cartan matrix, is rather written as $2 \delta_{a b}-G_{a b}$. One passes from one sign to the other by the change $\alpha_{a} \rightarrow(-1)^{(N-1) \tau(a)} \alpha_{a}$. This new convention has the merit of sparing a lot of irrelevant signs and of unifying the cases of $N$ even and odd. One should however recall this unorthodox choice of sign when examining the case $N=2$.

Note also that according to the usual conventions, all the non negative scalar products of roots in (29) should be represented by broken lines in the generalized Dynkin diagrams. For the ease of drawing, we use solid lines. 
The signature of the metric $g$ is of central importance. In particular it is well known that the group $\Gamma$ is of finite order if and only if the metric is positive definite. In the present case, one knows the eigenvalues of $g$

$$
g^{(\lambda)}=\sum_{p=0}^{N} \gamma_{p}^{(\lambda)}
$$

with the convention that $\gamma_{0}^{(\lambda)}=\gamma_{N}^{(\lambda)}=1$. Using (11), it takes the multiplicative form

$$
g^{(\lambda)}=\prod_{i=1}^{N}\left(1+e^{-\frac{2 i \pi}{h}\left(e_{i}, \lambda\right)}\right) .
$$

Then one has the following

Proposition 2.1 : The form $\langle$,$\rangle is positive definite if and only if the$ graph is of class II and

$$
\begin{array}{ll}
N=2 & \forall h \geq 3 \\
N=3 & h=4,5 \\
N \geq 4 & h=N+1 .
\end{array}
$$

The principle of the proof is to use the fact that the weight 0 in class I, or $\rho$ in class II, is known to belong to the set of exponents, together with its $\sigma$-orbit. Thus for class I,

$$
\gamma_{p}^{\left(\sigma^{\ell}(0)\right)}=e^{2 i \pi \frac{p \ell}{N}}\left(\begin{array}{l}
N \\
p
\end{array}\right)
$$

and

$$
g^{\left(\sigma^{\ell}(0)\right)}=\left(1+e^{2 i \pi \frac{\ell}{N}}\right)^{N}=(-1)^{\ell}\left(2 \cos \frac{\pi \ell}{N}\right)^{N}
$$

For $\ell=1$, this vanishes for $N=2$, and is negative for $N>2$. A similar discussion may be carried out for the class II, looking at the orbit of $\rho$. The details have been provided in [Z].

In that same reference [Z], the graphs which satisfy the conditions of Proposition 2 have been spelled out. For $N=2$, one recovers of course the $A D E$ Dynkin diagrams, together with the other CoxeterDynkin diagrams if the integrality of the matrix elements is relaxed. For $h=N+1$, the only graph is the fusion graph of $\widehat{s l}(N)$ at level $k=1$. At that level, the integrable weights are $\rho$ and the fundamental weights shifted by $\rho, \Lambda_{i}^{\prime}:=\Lambda_{i}+\rho, i=0, \cdots, N-1, \Lambda_{0}:=0$. The fusion ring of $\widehat{s l}(N)_{1}$ is isomorphic to the cyclic group $\mathbb{Z}_{N}$, with $G_{p}$ acting as a cyclic permutation of these weights, $\Lambda_{i}^{\prime} \mapsto \Lambda_{i+p \bmod N}^{\prime}$, and the graph of $G_{p}$ is 
thus a star-polygon. For $N=3$ and $h=5$, two solutions are known. The first has $n=3$ vertices, and all off diagonal entries of $G$ are $G_{a b}=2 \cos \frac{\pi}{5}$. The second has 6 vertices, it is the fusion graph of $\widehat{s l}(3)$ at level 2 . It is most likely that those are the only solutions $[\mathrm{Z}]$.

The form $\langle$,$\rangle is thus generically indefinite and the group of infinite$ order. In general, it may be proved that the number of negative eigenvalues of the bilinear form $g$ is even [Z]. Moreover, for graphs of class II, the number of zeros is also even. (See also a conjecture on the signature at the end of next subsection.) A few identifications or isomorphisms of such infinite groups have been obtained in reference [Z], to which we refer the reader. See also some results in sect. 4 below.

\subsection{Coxeter element}

The properties of the Coxeter element, product of all the reflections relative to simple roots of finite Coxeter groups, are well known [C]. There is a weak analogue in the more general class of reflection groups which we are considering here. One can make use of the grading of vertices to define the following product of reflections

$$
R=\prod_{\tau(a)=0} S_{a} \prod_{\tau(b)=1} S_{b} \cdots \prod_{\tau(f)=N-1} S_{f}
$$

Proposition 2.2 : The element $R$ is independent of the order of the $S$ within each block; it is conjugate in the linear group $G L(n)$ to the product $-T^{-1} T^{t}$ of the matrices defined at the end of sect. 1.4, and its spectrum is of the form

$$
(-1)^{N} \exp N \frac{-2 i \pi}{h}\left(e_{j}, \lambda\right) \quad \lambda \in \operatorname{Exp}, \quad \forall j \text { fixed: } 1 \leq j \leq N .
$$

(Indeed this set of numbers is independent of $j=1, \cdots, N$. ) The proof is a simple extension of the original proof by Coxeter [C]. It has been given in [Z] (up to changes of signs due to the changes of conventions in the metric), and it will be only sketched here. On the one hand, one proves that the $i$-th term in the product (37) equals

$$
\begin{aligned}
& \prod_{\tau(b)=i-1} S_{b}=\left(\begin{array}{cccccc}
\mathbf{1} & & & & & \\
& \ddots & & & & \\
& & \mathbf{1} & -A_{i i+1} & \cdots & -A_{i N} \\
& 0 & & \ddots & &
\end{array}\right)\left(\begin{array}{ccccc}
\mathbf{1} & & & & \\
& \ddots & & & \\
-A_{i 1} & \cdots & -\mathbf{1} & \\
& 0 & & \ddots & \\
& & & & \mathbf{1}
\end{array}\right) \\
& =: B_{i} C_{i}
\end{aligned}
$$


and that $B_{i}$ commutes with $C_{j}, 1 \leq j<i \leq N$. Since $B_{1} \cdots B_{N}=T^{-1}$ and $C_{1} \cdots C_{N}=-T^{t}$, the product $R$ is equal to the product $-T^{-1} T^{t}$. On the other hand, the polynomial with roots $-\varepsilon_{j}^{(\lambda)}=-\exp -\frac{2 i \pi}{h}\left(e_{j}, \lambda\right)$ reads

$$
\begin{aligned}
\Delta(z) & =\prod_{j=1}^{N} \prod_{\lambda \in \operatorname{Exp}}\left(z+\varepsilon_{j}^{(\lambda)}\right) \\
& =\operatorname{det}\left(z^{N} \mathbf{1}+z^{N-1} G_{1}+z^{N-2} G_{2}+\cdots+z G_{N-1}+\mathbf{1}\right) \\
& =\operatorname{det}\left(\begin{array}{ccccc}
\left(z^{N}+1\right) \mathbf{1} & z^{N-1} A_{12} & z^{N-2} A_{13} & \cdots & z A_{1 N} \\
z A_{21} & \left(z^{N}+1\right) \mathbf{1} & z^{N-1} A_{23} & & z^{2} A_{2 N} \\
\vdots & & & \ddots & \\
z^{N-1} A_{N 1} & z^{N-2} A_{N 2} & \cdots & & \left(z^{N}+1\right) \mathbf{1}
\end{array}\right) \\
& =\operatorname{det}\left(z^{N} T+T^{t}\right)=\operatorname{det}\left(z^{N} \mathbf{1}-\left(-T^{-1} T^{t}\right)\right),
\end{aligned}
$$

using (11) and (25). This proves that the eigenvalues of $R=-T^{-1} T^{t}$ are the $\left(-\varepsilon_{j}(\lambda)\right)^{N}$, which are indeed independent of $j$.

It is an interesting question to know if this Coxeter element is of finite or infinite order. Even though its eigenvalues are roots of unity, $R$ may be non-diagonalizable and thus of infinite order. One has the following

Proposition 2.3 : For any graph of class $I$, the Coxeter element $R$ is of infinite order.

The obstruction to diagonalizability of $R$ comes from its eigenvalue $(-1)^{N}$, which is associated by Proposition 2.2 with the exponent 0 and its $\sigma$ orbit, and which has thus a multiplicity at least equal to $N$. The proof consists in finding a subspace of $V$ in which $R$ takes a triangular form with this eigenvalue on the diagonal. Let

$$
\beta_{i}=\sum_{a: \tau(a)=i} \psi_{a}^{(0)} \alpha_{a}, \quad i=0, \cdots, N-1
$$

and

$$
\delta_{k}=\sum_{i=k}^{N-1}(-1)^{i-k}\left(\begin{array}{l}
i \\
k
\end{array}\right) \beta_{i}, \quad k=0, \cdots, N-1
$$

for $i, k=0, \cdots, N-1$. Then denote $R_{j}:=\prod_{\tau(a)=j} S_{a}$. One computes 
easily, using the eigenvalues $\gamma_{p}^{(0)}$ of (12),

$$
\begin{aligned}
& R_{j} \beta_{i}=\beta_{i}-\left(\begin{array}{c}
N \\
|i-j|
\end{array}\right) \beta_{j}-\delta_{i j} \beta_{i} \\
& R_{j} \delta_{k}=\delta_{k}-(-1)^{k}\left[(-1)^{i}\left(\begin{array}{l}
j \\
k
\end{array}\right)+\sum_{i=0}^{N-1}(-1)^{i}\left(\begin{array}{l}
i \\
k
\end{array}\right)\left(\begin{array}{c}
N \\
|i-j|
\end{array}\right)\right] \beta_{j}
\end{aligned}
$$

Then it is tedious but straightforward to establish by induction that

$$
\begin{aligned}
R_{j} R_{j-1} \cdots R_{0} \delta_{k}=\delta_{k} & -\sum_{i=0}^{j-k}(-1)^{i}\left(\begin{array}{c}
i+k \\
k
\end{array}\right) \beta_{i+k} \\
& +(-1)^{N-k} \sum_{i=0}^{j}\left(\left(\begin{array}{c}
N+i \\
k
\end{array}\right)-\sum_{\ell=j+1}^{k}\left(\begin{array}{c}
i \\
\ell
\end{array}\right)\left(\begin{array}{c}
N \\
k-\ell
\end{array}\right)\right) \beta_{i}
\end{aligned}
$$

in which the sum $\sum_{\ell=j+1}^{k}\left(\operatorname{resp} \sum_{i=0}^{j-k}\right)$ reduces to naught for $j \geq k$ (resp $k \geq j$ ). Taking $j=N-1$ in (44), it follows that

$$
\begin{aligned}
R^{-1} \delta_{k}=R_{N-1} \cdots R_{0} \delta_{k} & =(-1)^{N-k} \sum_{i=0}^{N-1}\left(\begin{array}{c}
N+i \\
k
\end{array}\right) \beta_{i} \\
& =(-1)^{N}\left(\delta_{k}+\sum_{i=1}^{k}(-1)^{i}\left(\begin{array}{c}
N \\
i
\end{array}\right) \delta_{k-i}\right)
\end{aligned}
$$

This is the triangular form referred to above and this clearly contradicts the possibility that $R$ be of finite order. Note that the previous proof is in essence the generalization to $N$-colourable graphs of the proof by Berman, Lee and Moody for bi-partite graphs [BLM].

In contrast with this case of the class I, it appears that

Proposition 2.4 : For any graph of class II, the Coxeter element is of finite order.

This has been proved by V. Petkova using a different approach that extends the discussion of Kostant $[\mathrm{K}]$ and that also yields the result of Proposition $2.3[\mathrm{Pe}]$.

If the Coxeter element $R$ is of finite order, this order is equal at most to $h$ if $N h$ is even and to $2 h$ if $N h$ is odd. The order may be smaller, e.g. for the graphs $\mathcal{D}^{(6)}$ and $\mathcal{D}^{(9)}$ of Fig. 3, it is 2 resp. 6, while the value of $h$ is given by the superscript. If $m$ is a common divisor of $N$ and $h$ such that $\forall \lambda \in \operatorname{Exp}, \tau(\lambda)=0 \bmod m$, then the order of $R$ is $h / m$ (resp $2 h / m)$ if $N(N-1+h) / m$ is even (resp odd) [Pe].

The ordering chosen in (37) is the only natural one for most cases. In the case of fusion graphs, however, it is possible to use a lexicographic ordering of the vertices, namely of the integrable weights $\lambda$ at some 
level $k$ of $\widehat{s l}(N)_{k}$. Thus for $\lambda, \mu \in \mathcal{P}_{++}^{(h)}$ with the notations of equ (1), one writes $\lambda \prec \mu$ if $\lambda_{N-1}=\mu_{N-1}, \cdots \lambda_{p}=\mu_{p}$ and $\lambda_{p-1}<\mu_{p-1}$. One verifies for the lowest levels, and it is quite likely to be true in general, that the product of the $S$ 's according to this ordering defines another Coxeter element equivalent to (37). This definition of the Coxeter element is more natural from the point of view of the monodromy of the associated singularity (see below sect.4).

The eigenvalues of the Coxeter element as given by Prop. 2.2 seem to specify the signature of the metric, in the case of class II graphs. For each exponent of $\lambda$ define the real numbers

$$
\begin{aligned}
q_{\lambda}^{(\mathrm{R})} & =-\frac{N}{h}\left(e_{N}, \lambda\right)-\frac{N-1}{2} \\
& =\frac{1}{h} \sum j\left(\lambda_{j}-1\right)+\frac{(N-h)(N-1)}{2 h} .
\end{aligned}
$$

Note that the eigenvalues of $-R$ are $\exp 2 i \pi q_{\lambda}^{(\mathrm{R})}$, for $\lambda \in \operatorname{Exp}$. Then

Conjecture 2.5 : The signature of the metric $g$ for class II graphs is $(r+, s-, t 0)$ where $r$ is the number of $q_{\lambda}^{(\mathrm{R})}$ which fall in an interval ] $2 p-\frac{1}{2}, 2 p+\frac{1}{2}[, p \in \mathbb{Z}, s$ the number of those in an interval $] 2 p+\frac{1}{2}, 2 p+\frac{3}{2}[$, and $t=n-r-s$ the number of those which are half-integers.

Alternatively, the signature is obtained by looking at the signs of Re $\exp i \pi q_{\lambda}^{(R)}$. This is suggested by the work of Steenbrink [St] on the intersection form of quasi-homogeneous singularities and by the one of Cecotti and Vafa $[\mathrm{CV}]$ on $\mathcal{N}=2$ supersymmetric field theories and

topological field theories. In the latter, $q_{\lambda}^{(\mathrm{R})}$ are the $U(1)$ charges of the Ramond ground states. Cecotti and Vafa study the monodromy operator of a linear system related to the $t t^{*}$ equations. The previous conjecture follows from their work if one identifies their monodromy operator with the present Coxeter element, which seems highly plausible. The last part of the conjecture is easy to prove: using once again eq. (21) and the product formula (34) for $g^{(\lambda)}$, one proves that on each $\sigma$-orbit of an exponent $\lambda$, there are as many vanishing $g^{\left(\lambda^{\prime}\right)}$ as there are $\varepsilon_{N}\left(\lambda^{\prime \prime}\right)^{N}$ equal to $(-1)^{N}$, i.e. half-integral $q_{\lambda^{\prime \prime}}^{(R)}$.

Conjecture 2.5 has been tested on all known examples.

\section{Pasquier algebras, subalgebras and folding.}

\subsection{Pasquier algebras and (quasi) C-algebras}

For any graph of the previous type, the eigenvector $\psi^{(0)}$ for the class I, or $\psi^{(\rho)}$ for class II, is the eigenvector of $G_{1}$ of largest eigenvalue, i.e. the 
so-called Perron-Frobenius eigenvector. As the graph is connected (cf axiom 2), all the components of the Perron-Frobenius eigenvector are non-vanishing and may be chosen real positive. We shall denote $\Phi$ this special exponent 0 (class I) resp $\rho$ (class II).

Now consider the numbers $[\mathrm{P}]$

$$
M_{\lambda \mu}{ }^{\nu}=\sum_{a} \frac{\psi_{a}^{(\mu)} \psi_{a}^{(\nu)} \psi_{a}^{(\nu *)}}{\psi_{a}^{(\Phi)}}
$$

Since the $\psi$ are orthonormal, $M_{\Phi \mu}{ }^{\nu}=\delta_{\mu \nu}$. Since they satisfy $\psi_{a}^{(\lambda) *}=$ $\psi_{a}^{(\bar{\lambda})}=\psi_{\bar{a}}^{(\lambda)}$, the $M_{\lambda \mu}^{\nu}$ are real, the involution in Exp: $\lambda \mapsto \bar{\lambda}$ is an automorphism $M_{\lambda \mu}{ }^{\nu}=M_{\bar{\lambda} \bar{\mu}}{ }^{\bar{\nu}}$, and

$$
\begin{aligned}
M_{\lambda \mu}{ }^{\Phi} & =\sum_{a} \psi_{a}^{(\lambda)} \psi_{a}^{(\mu)} \\
& =\sum_{a} \psi_{a}^{(\lambda)} \psi_{a}^{(\bar{\mu}) *}=\delta_{\lambda \bar{\mu}}
\end{aligned}
$$

The algebra over $\mathbb{C}$ defined by these structure constants $M_{\lambda \mu}{ }^{\nu}$ is a commutative and associative (as is readily verified) algebra, called the Pasquier algebra relative to the graph. It is a quasi-C-algebra in the following sense:

Definition : An algebra $\mathcal{A}$ over $\mathbb{C}$ with a given basis $x_{1}, \cdots, x_{n}$, is a quasi $C$-algebra if it satisfies the following axioms:

i) it is a commutative and associative algebra with real structure constants $p_{\alpha \beta}^{\gamma}$, i.e. $x_{\alpha} \cdot x_{\beta}=\sum_{\gamma} p_{\alpha \beta}^{\gamma} x_{\gamma}$

ii) it has an identity element, denoted $x_{1}$, i.e. $p_{1 \alpha}{ }^{\beta}=\delta_{\alpha \beta}$;

iii) there is an involution on the generators $x_{\alpha} \mapsto x_{\bar{\alpha}}$ which is an automorphism of the algebra, i.e. $p_{\alpha \beta}^{\gamma}=p_{\bar{\alpha} \bar{\beta}} \overline{\bar{\gamma}}$;

iv) $p_{\alpha \beta}{ }^{1}=k_{\alpha} \delta_{\alpha \bar{\beta}}$, with $k_{\alpha}$ a real positive number: $k_{\alpha}>0$.

Definition : $[\mathrm{BI}]$ A $C$-algebra is a quasi C-algebra which satisfies the additional property

v) the $k_{\alpha}$ form a one-dimensional representation of the algebra.

The theory developed by Bannai and Ito may be summarized as follows. The axioms i-iv) imply that a quasi-C-algebra is semi-simple. Let $e_{i}, i=1, \cdots, n$ denote the idempotents $e_{i} \cdot e_{j}=\delta_{i j} e_{i}$ and decompose

$$
x_{\alpha}=\sum_{i} p_{\alpha}(i) e_{i}
$$

If axiom v) is also satisfied, one may choose $p_{\alpha}(1)=k_{\alpha}$. Then one may introduce a second multiplication on $\mathcal{A}$ for which the original generators 
act as idempotents $x_{\alpha} \circ x_{\beta}=\delta_{a b} x_{a}$. By inversion of (49) this yields a non trivial $e_{i} \circ e_{j}=q_{i j}{ }^{k} e_{k}$. $\mathcal{A}$ is also a C-algebra for this second multiplication, and there are thus two dual $\mathrm{C}$-algebra structures defined on the set $\mathcal{A}$.

One has a nice illustration of this in the present context of graphs. It is clear that the axioms of a quasi C-algebra are satisfied by the $M_{\lambda \mu}{ }^{\nu}$. On the other hand, it is easy to verify that one knows $n$ 1-dimensional representations of the algebra, namely

$$
\sum_{\nu} M_{\lambda \mu}{ }^{\nu} \frac{\psi_{a}^{(\nu)}}{\psi_{a}^{(\Phi)}}=\frac{\psi_{a}^{(\lambda)}}{\psi_{a}^{(\Phi)}} \frac{\psi_{a}^{(\mu)}}{\psi_{a}^{(\Phi)}} .
$$

Thus if there exists one vertex, call it 1 , such that $\psi_{1}^{(\lambda)}$ are non vanishing for all $\lambda \in \operatorname{Exp}$, then at the possible price of an overall change of sign one may assume them positive and

$$
\sqrt{k_{\lambda}}:=\frac{\psi_{1}^{(\lambda)}}{\psi_{1}^{(\Phi)}}
$$

and $p_{\lambda \mu}^{\nu}=\sqrt{\frac{k_{\lambda} k_{\mu}}{k_{\nu}}} M_{\lambda \mu}{ }^{\nu}$ satisfy all the conditions of a C-algebra, including v). The quantities $\frac{\psi_{a}^{(\lambda)}}{\psi_{1}^{(\lambda)}}$ are the one-dimensional representations of a dual algebra with structure constants

$$
N_{a b}^{c}=\sum_{\lambda \in \operatorname{Exp}} \frac{\psi_{a}^{(\lambda)} \psi_{b}^{(\lambda)} \psi_{c}^{(\lambda) *}}{\psi_{1}^{(\lambda)}} .
$$

The algebra $\mathcal{A}^{*}$ with structure constants $q_{a b}{ }^{c}=\sqrt{k_{a}^{*} k_{b}^{*} / k_{c}^{*}} N_{a b}{ }^{c}$, with $\sqrt{k_{a}^{*}}=\psi_{a}^{(\Phi)} / \psi_{1}^{(\Phi)}$, is a C-algebra, and $\mathcal{A}$ and $\mathcal{A}^{*}$ form a pair of dual Calgebras. The most favorable case is when the structure constants of both algebras are non negative (see below).

In fact the three possibilities occur in our graphs :

1. For a given choice of the $\psi$, non existence of a vertex $a$ such that $\forall \lambda, \quad \psi_{a}^{(\lambda)}>0$. This is for example the case of the $\mathcal{D}^{(9)}$ diagram of Fig. 3 if we take for the exponent $\lambda=(3,3)$ eigenvectors with components on the three rightmost vertices equal respectively to $(0,0,0),\left(1, \omega, \omega^{2}\right) / \sqrt{3}$ and $\left(1, \omega^{2}, \omega\right) / \sqrt{3}$, with $\omega=\exp \frac{2 i \pi}{3}$.

2. Existence of such a vertex, but the resulting $M$ and $N$ structure constants are of either sign. This is for example the case of the $D_{2 \ell+1}$ or $E_{7}$ Dynkin diagrams. This is also the case of the graph of Fig. 1(b).

3. Existence of such a vertex, and the $M$ and $N$ (and $p$ and $q$ ) structure constants are non negative. This is the case of all graphs of 
class I obtained from a finite subgroup of $S U(N)$, since the two $M$ and $N$ algebras are then isomorphic to the class and the character algebras respectively and their structure constants are thus integers in appropriate bases. This is also (by inspection) the case of the $A_{n}$, $E_{6}$ and $E_{8}$ Dynkin diagrams, as well as the $D_{2 \ell}$ ones, if a suitable choice is made for the eigenvectors corresponding to the exponent $\lambda=2 \ell-1$. This is also the case of the fusion graphs of $\widehat{s l}(N)$ at any level, since in that case the $M$ and $N$ algebras are both isomorphic to the fusion algebra, and the structure constants are the non negative fusion coefficients.

Remark: A drawback of our approach is to rely on the choice of an explicit basis of the C-algebra(s), i.e. of the eigenvectors $\psi$. When some exponents have multiplicities, there are several choices of the $\psi$ 's. Properties like the existence of the vertex 1, or the positivity of the $M$ and/or $N$ algebras depend on this choice.

\subsection{Subalgebras}

Suppose that the algebra defined by the structure constants $M$, the $M$ algebra in short, admits a sub-algebra in the following sense:

There is a subset $\hat{T}$ of the set of exponents such that

$$
\lambda, \mu \in \hat{T} \quad M_{\lambda \mu}{ }^{\nu} \neq 0 \Rightarrow \nu \in \hat{T}
$$

We also assume that the set $\hat{T}$ is stable under conjugation $\lambda \mapsto \bar{\lambda}$ and under the action of the $\mathbb{Z}_{N}$ automorphism $\sigma$. Note that the stability under conjugation implies that the identity (denoted $\Phi$ ) belongs to the set $\hat{T}$. The stability under conjugation is itself satisfied if the $M$ algebra is a C-algebra (see the proof in $[\mathrm{BI}]$ ). On the other hand, the assumption of stability under $\sigma$ is a non trivial condition, which eliminates some possibilities. For example in the case $E_{6}$, the subset $\{1,7\}$ of exponents defines a sub-algebra, but is not stable under $\sigma: \lambda \mapsto 12-\lambda$.

We now make the further assumption that the subalgebra defined by $\hat{T}$ has the following property

Property $\wp$ : There exists a partition of the set of vertices $\mathcal{V}=\bigcup_{i} T_{i}$ into classes such that

i) if $\lambda \in \hat{T}, \psi_{a}^{(\lambda)} / \psi_{a}^{(1)}$ is independent of the representative $a$ within the class $T_{i}$

ii) if $\lambda \notin \hat{T}$, then for any class $T_{i}, \sum_{b \in T_{i}} \psi_{b}^{(\lambda)} \psi_{b}^{(\Phi)}=0$.

Both conditions may be conveniently embodied in a single relation

$$
\forall \lambda, \forall T_{i}, \forall a \in T_{i} \quad \sum_{b \in T_{i}} \psi_{b}^{(\lambda)} \psi_{b}^{(\Phi)}=\delta_{\lambda \in \hat{T}} \frac{\psi_{a}^{(\lambda)}}{\psi_{a}^{(\Phi)}} \sum_{b \in T_{i}}\left(\psi_{b}^{(\Phi)}\right)^{2}
$$


Before looking at the implications of that property, let us first show that it is natural. One knows at least three situations in which it is realized.

Proposition 3.1 Property $\wp$ is verified in either of the three following cases

1) The subset $\hat{T}$ is the $\sigma$ orbit of $\Phi$.

2) The subset $\hat{T}$ is associated with a $\mathbb{Z}_{r}$ symmetry of the graph (in a sense to be explained).

3) The Pasquier algebra defines a $C$-algebra and the $M$ and $N$ structure constants are non negative.

First note that these cases are not exclusive of one another, as we shall see. Also, we have no claim that they exhaust all the situations where property $\wp$ is satisfied.

1) This is the most trivial case: the subset $\hat{T}=\left\{\sigma^{\ell}(\Phi) ; \ell=0, \cdots, N-1\right\}$ gives rise to a subalgebra, as follows from (20b): if $\lambda, \mu \in \hat{T}, \lambda=\sigma^{\ell}(\Phi)$, $\mu=\sigma^{m}(\Phi)$, thus

$$
M_{\lambda \mu}^{\nu}=\sum_{a} e^{\frac{2 i \pi}{N}(\ell+m) \tau(a)} \psi_{a}^{(\Phi)} \psi_{a}^{(\nu) *}=\delta_{\nu, \sigma^{\ell+m}(\Phi)}
$$

which is non vanishing only if $\nu \in \hat{T}$. Then the classes $T_{i}$ contain all vertices with the same grading

$$
T_{i}=\{a \in \mathcal{V}: \tau(a)=i\} \quad i=1, \cdots, N
$$

Let us show that $\wp$ is fulfilled. Use the orthogonality of the $\psi$ 's and relation $(20 b)$ to write

$$
\begin{aligned}
\sum_{b} \psi_{b}^{(\lambda)} \psi_{b}^{\left(\sigma^{\ell}(\mathbb{\Phi})\right) *} & =\delta_{\lambda, \sigma^{\ell}(\mathbf{\Phi})} \\
& =\sum_{b} \psi_{b}^{(\lambda)} \psi_{b}^{(\Phi) *} e^{-2 i \pi \frac{\ell \tau(b)}{N}} \\
& =\sum_{T_{j}} \sum_{b \in T_{j}} \psi_{b}^{(\lambda)} \psi_{b}^{(\Phi) *} e^{-2 i \pi \frac{j \ell}{N}}
\end{aligned}
$$

Multiplying by $\exp 2 i \pi \frac{\ell J}{N}$ and summing over $\ell$ from 0 to $N-1$ projects onto $j=J$, giving

$$
\sum_{b \in T_{J}} \psi_{b}^{(\lambda)} \psi_{b}^{(\Phi) *}=\frac{1}{N} \sum_{\ell=0}^{N-1} e^{2 i \pi \frac{J \ell}{N}} \delta_{\lambda, \sigma^{\ell}(\Phi)} .
$$

But $\forall a \in T_{J}$, if $\lambda=\sigma^{\ell}(\Phi), \psi_{a}^{(\lambda)} / \psi_{a}^{(\Phi)}=e^{2 i \pi \frac{J \ell}{N}}$. We also use the fact that $\sum_{b \in T_{J}}\left|\psi_{b}^{(\Phi)}\right|^{2}$ is independent of $J=0,1, \cdots, N-1$ and thus equal to $1 / N$. 
This follows simply from the equality [PZ]

$$
\sum_{a \in T_{J}, b} \psi_{a}^{(\Phi)}\left(G_{1}\right)_{a b} \psi_{b}^{(\Phi) *}=\gamma^{(\Phi)} \sum_{a \in T_{J}}\left|\psi_{a}^{(\Phi)}\right|^{2}=\gamma^{(\Phi)} \sum_{b \in T_{J+1}}\left|\psi_{b}^{(\Phi)}\right|^{2}
$$

Putting everything together, we find that (56) amounts to relation (54).

2) Suppose that the graph admits a $\mathbb{Z}_{r}$ automorphism, i.e. there a bijection between vertices $a \mapsto \varphi(a), \varphi^{r}=i d$, which preserves the grading $\tau(\varphi(a))=\tau(a)$ and the adjacency matrix $G_{\varphi(a) \varphi(b)}=G_{a b}$. One may then diagonalize $G$ (and all $G_{p}$ ) by means of eigenvectors satisfying

$$
\psi_{\varphi(a)}^{(\lambda)}=e^{2 i \pi \frac{q}{r}} \psi_{a}^{(\lambda)}
$$

i.e. of a given "charge" $q=q(\lambda) \in \mathbb{Z}_{r}$. Note that $\psi^{(\Phi)}$, whose components are all positive, is necessarily of zero charge and that

$$
M_{\lambda \mu}^{\nu}=e^{-2 i \pi \frac{q(\lambda)+q(\mu)-q(\nu)}{r}} M_{\lambda \mu}^{\nu}
$$

does not vanish only if $q(\nu)=q(\lambda)+q(\mu) \bmod N$. In particular a subalgebra of the $M$ algebra is provided by the set of exponents

$$
\hat{T}=\{\lambda \mid q(\lambda)=0\}
$$

In that case, the classes of vertices $T_{i}$ are the $\varphi$-orbits

$$
\text { class of } a=T_{i}=\left\{\varphi^{j}(a), j=0, \cdots, r-1 .\right\}
$$

The length $\ell$ of the orbit may be smaller than $r$, it is a divisor of $r$; $\varphi^{\ell}(a)=a$ implies that the only non vanishing $\psi_{a}^{(\lambda)}$ are those for which $q(\lambda)$ is a multiple of $\frac{r}{\ell}$. One then computes easily, if $a \in T_{i}$ belongs to an orbit of length $\ell$ of $\varphi$

$$
\begin{aligned}
\sum_{b \in T_{i}} \psi_{b}^{(\lambda)} \psi_{b}^{(\Phi)} & =\sum_{j=0}^{\ell-1} \psi_{\varphi^{j}(a)}^{(\lambda)} \psi_{\varphi^{j}(a)}^{(\Phi)} \\
& =\left(\sum_{j=0}^{\ell-1} e^{2 i \pi \frac{q(\lambda) j}{r}}\right) \frac{\psi_{a}^{(\lambda)}}{\psi_{a}^{(\Phi)}}\left(\psi_{a}^{(\Phi)}\right)^{2} \\
& =\left\{\begin{array}{lll}
\ell \frac{\psi_{a}^{(\lambda)}}{\psi_{a}^{(\Phi)}}\left(\psi_{a}^{(\Phi)}\right)^{2}=\frac{\psi_{a}^{(\lambda)}}{\psi_{a}^{(\Phi)}} \sum_{b \in T_{i}}\left(\psi_{b}^{(\Phi)}\right)^{2} & \text { if } \quad q(\lambda)=0 \quad \bmod r \\
0 & \text { otherwise . }
\end{array}\right.
\end{aligned}
$$

This completes the proof that case 2) satisfies property $\wp$. 
3) In the case of a C-algebra with non negative $M$ and $N$ (and thus $p$ and $q$ ) structure constants, one may apply the theorem of Bannai and Ito ([BI], theorem 9.9). $\hat{T}$ defines a C-subalgebra. Then one defines the equivalence relation between exponents $\mu \sim \nu$ if $\exists \lambda \in \hat{T}: M_{\lambda \mu}{ }^{\nu} \neq 0$. Thus there is a partition of the set $\operatorname{Exp}=\cup_{\alpha} \hat{T}_{\alpha}$ into equivalence classes. One then proves that there exists a subset $T$ of the dual set $\mathcal{V}$ such that

$$
\forall a, \forall \hat{T}_{\alpha}, \forall \lambda \in \hat{T}_{\alpha} \quad \sum_{\mu \in \hat{T}_{\alpha}} \psi_{a}^{(\mu)} \psi_{1}^{(\mu)}=\delta_{a \in T} \frac{\psi_{a}^{(\lambda)}}{\psi_{1}^{(\lambda)}} \sum_{\mu \in \hat{T}_{\alpha}}\left(\psi_{1}^{(\mu)}\right)^{2}
$$

(which is the dual form of (54)). This subset $T$ defines in turn a subalgebra of the dual $N$ algebra, in the sense that $a, b \in T, N_{a b}{ }^{c} \neq 0 \Rightarrow c \in T$, and relation (54) is itself satisfied.

Consequences of property $\wp$

1. Let $\mathcal{N}_{i}$ be the real positive numbers defined by

$$
\mathcal{N}_{i}^{-2}=\sum_{a \in T_{i}}\left(\psi_{a}^{(\Phi)}\right)^{2}
$$

Then for any $\lambda \in \hat{T}$, define

$$
\Psi_{i}^{(\lambda)}=\mathcal{N}_{i}^{-1} \frac{\psi_{a}^{(\lambda)}}{\psi_{a}^{(\Phi)}}
$$

By virtue of relation (54), it is independent of $a \in T_{i}$ and in fact

$$
\Psi_{i}^{(\lambda)}=\mathcal{N}_{i} \sum_{b \in T_{i}} \psi_{b}^{(\lambda)} \psi_{b}^{(\Phi)}
$$

Corollary 3.2 1. The $\Psi_{i}^{(\lambda)}$, as $i$ runs over the classes $T_{i}$ and $\lambda$ over the subset $\hat{T}$, form an orthogonal and complete system of rank equal to the cardinality of $\hat{T}$.

$$
\begin{aligned}
& \sum_{\lambda \in \hat{T}} \Psi_{i}^{(\lambda)} \Psi_{j}^{(\lambda) *}=\delta_{i j} \\
& \sum_{i} \Psi_{i}^{(\lambda)} \Psi_{i}^{(\mu) *}=\delta_{\lambda \mu} \quad \text { for } \quad \lambda, \mu \in \hat{T}
\end{aligned}
$$

Thus the number of classes $T_{i}$ equals the cardinality of $\hat{T}$ (i.e. the dimension of the $M$ subalgebra).

2. The subalgebra $M_{\lambda \mu}{ }^{\nu}, \lambda, \mu, \nu \in \hat{T}$, is diagonal in that basis

$$
M_{\lambda \mu}{ }^{\nu}=\sum_{i} \frac{\Psi_{i}^{(\lambda)} \Psi_{i}^{(\mu)} \Psi_{i}^{(\nu) *}}{\Psi_{i}^{(\Phi)}} .
$$


Proof: the proof of the three statements $(67 a-c)$ follows the same line and makes use of (54). Let us present it for (67b) only

$$
\begin{aligned}
\sum_{i} \Psi_{i}^{(\lambda)} \Psi_{i}^{(\mu) *} & =\sum_{i} \mathcal{N}_{i}^{-2} \frac{\psi_{a}^{(\lambda)}}{\psi_{a}^{(\Phi)}} \frac{\psi_{a}^{(\mu) *}}{\psi_{a}^{(\Phi)}}=\sum_{i} \frac{\psi_{a}^{(\lambda)}}{\psi_{a}^{(\Phi)}} \sum_{b \in T_{i}} \psi_{b}^{(\mu) *} \psi_{b}^{(\Phi)} \quad \forall a \in T_{i} \\
& =\sum_{i} \sum_{b \in T_{i}} \frac{\psi_{b}^{(\lambda)}}{\psi_{b}^{(\Phi)}} \psi_{b}^{(\mu) *} \psi_{b}^{(\Phi)}=\sum_{b} \psi_{b}^{(\lambda)} \psi_{b}^{(\mu) *}=\delta_{\lambda \mu} .
\end{aligned}
$$

2. We now use the assumption of stability of $\hat{T}$ under $\sigma$ to prove the

Corollary 3.3 All the vertices within a given class $T_{i}$ have the same grading $\tau$.

Proof: Since $\Phi \in \hat{T}$, let us evaluate relation (54) for $\lambda=\sigma(\Phi)$.

$$
\sum_{b \in T_{i}} \psi_{b}^{(\sigma(\Phi))} \psi_{b}^{(\Phi)}=\frac{\psi_{a}^{(\sigma(\Phi))}}{\psi_{a}^{(\Phi)}} \sum_{b \in T_{i}}\left(\psi_{b}^{(\Phi)}\right)^{2}
$$

thus, using (20b)

$$
\sum_{b \in T_{i}}\left(\psi_{b}^{(\Phi)}\right)^{2} e^{2 i \pi \frac{\tau(b)-\tau(a)}{N}}=\sum_{b \in T_{i}}\left(\psi_{b}^{(\Phi)}\right)^{2}
$$

which is impossible if the phases are not equal to $0(\bmod 2 \pi)$. (Geometrically the module of the l.h.s. is the end-to-end length of a broken line, the r.h.s. the same when the line made of the same segments is straight!).

\subsection{Associated subgroup of the reflection group}

We now prove that with any $M$ subalgebra satisfying the previous property $\wp$, we may associate a subgroup of the reflection group $\Gamma$. As before, let $T_{i}$ denote the classes in the partition of $\mathcal{V}$.

Proposition 3.4 The operators $R_{i}=\prod_{a \in T_{i}} S_{a}, i=1, \cdots,|\hat{T}|$ generate a subgroup $\Gamma^{\prime}$ of the reflection group $\Gamma$. $R_{i}$ is itself a reflection in the hyperplane orthogonal to

$$
\beta_{i}=\mathcal{N}_{i} \sum_{a \in T_{i}} \psi_{a}^{(\Phi)} \alpha_{a}
$$

(with $\mathcal{N}_{i}$ defined in (64)). 
Thus $\Gamma^{\prime}$ is itself a reflection group in a subspace of dimension $|\hat{T}|$ of the space $V$, generated by the $\beta$. The partition into $T_{i}$ of the set of vertices provides the folding of the original "Dynkin diagram": all the vertices $a \in T_{i}$ are mapped onto a single vertex $i$ of the folded diagram, with the new "roots" $\beta_{i}$ given by the real linear combinations (71). The new set of vertices inherits the $\tau$ grading of the original ones, thanks to Corollary 3.3.

Proof of Proposition: By Corollary 3.3, all $a$ within $T_{i}$ have the same grading $\tau$ and thus the corresponding roots $\alpha_{a}$ are mutually orthogonal. Consider the product $\prod_{a \in T_{i}} S_{a}$. Its action on an arbitrary vector of $V$ reads

$$
\prod_{a \in T_{i}} S_{a} x=x-\sum_{a \in T_{i}}\left\langle\alpha_{a}, x\right\rangle \alpha_{a}
$$

Compare it to the reflection $R_{i}$ in an hyperplane (through the origin) orthogonal to the vector $\beta_{i}=\sum_{a \in T_{i}} B_{i a} \alpha_{a}$, with real coefficients $B_{i a}$. If $\beta_{i}$ is normed to $\left\langle\beta_{i}, \beta_{i}\right\rangle=2$,

$$
\begin{aligned}
R_{i} x & =x-\left\langle\beta_{i}, x\right\rangle \beta_{i} \\
& =x-\sum_{a, b \in T_{i}} B_{i a} B_{i b}\left\langle\alpha_{b}, x\right\rangle \alpha_{a} .
\end{aligned}
$$

$R_{i}$ and $\prod S_{a}$ have the same action in the subspace $\mathcal{E}_{i}$

$$
\mathcal{E}_{i}=\left\{x \mid \forall a \in T_{i} \quad \sum_{b \in T_{i}}\left(\alpha_{b}, x\right) B_{i b} B_{i a}=\left(\alpha_{a}, x\right)\right\}
$$

The different $R_{i}$ act in a consistent way in the space $\mathcal{E}=\cap_{i} \mathcal{E}_{i}$ provided each $\beta_{i}$ belongs to $\mathcal{E}$, i.e.

$$
\forall i, \forall a \in T_{i}, \forall j \quad \sum_{\substack{b \in T_{i} \\ c \in T_{j}}} B_{i a} B_{i b} B_{j c} g_{b c}=\sum_{c \in T_{j}} B_{j c} g_{a c} .
$$

This condition is fulfilled by $B_{i a}=\mathcal{N}_{i} \psi_{a}^{(\text {() }}$, i.e. $\beta_{i}$ as in Prop. 3.4. The normalisation factor ensures that $\left\langle\beta_{i}, \beta_{i}\right\rangle=2$. To check eq. (75), one diagonalizes the metric $g_{a c}=\sum_{\lambda \in \operatorname{Exp}} g^{(\lambda)} \psi_{a}^{(\lambda)} \psi_{c}^{(\lambda) *}$. Then a short calculation shows that both sides of (75) are equal to

$$
\sum_{\lambda \in \hat{T}} g^{(\lambda)} \psi_{a}^{(\lambda)} \frac{\psi_{c}^{(\lambda) *}}{\psi_{c}^{(\Phi)}}
$$

This completes the proof of Prop. 3.4. 
Note that the $\Psi_{i}^{(\lambda)}, \lambda \in \hat{T}$, defined in (65) diagonalize the new metric:

$$
\begin{aligned}
g_{i j} & =\left(\beta_{i}, \beta_{j}\right)=\mathcal{N}_{i}^{-1} \mathcal{N}_{j}^{-1} \sum_{\lambda \in \hat{T}} g^{(\lambda)} \frac{\psi_{a}^{(\lambda)}}{\psi_{a}^{(\Phi)}} \frac{\psi_{b}^{(\lambda) *}}{\psi_{b}^{(())}} \quad \forall a \in T_{i}, \forall b \in T_{j} \\
& =\sum_{\lambda \in \hat{T}} g^{(\lambda)} \Psi_{i}^{(\lambda)} \Psi_{j}^{(\lambda) *} .
\end{aligned}
$$

The geometry of the new root system $\left\{\beta_{i}\right\}$ is encoded into a graph which satisfies all axioms of sect. 1.2. From the metric (76) one derives the adjacency matrices $G_{i j}=g_{i j}-2 \delta_{i j}$ and $\left(G_{p}\right)_{i j}$, since the color $\tau(i)$ of each vertex is known (see (29), (14) and the remark at the end of sect 1.2). A determination (up to a common factor) of the Coxeter number and exponents of the folded graph is provided by

$$
h_{\text {folded }}=h, \quad \operatorname{Exp}_{\text {folded }}=\hat{T} .
$$

\subsection{A guided tour of the zoo}

There are a few cases which we may discuss in full generality. As we have seen (case 1 of Prop. 3.1), for any graph, the $M$ algebra admits a subalgebra associated with the exponents which are on the orbit of 1

$$
\hat{T}=\left\{\sigma^{\ell}(\Phi) ; \ell=0, \cdots, N-1\right\} .
$$

The graph may be folded in a trivial way, in which all vertices with the same color $\tau(a)=i, i=0, \cdots, N-1$ are mapped onto the same vertex $i+1$ of the folded graph. The scalar products of the new roots are also easy to compute. For $i \neq j$ :

$$
\begin{aligned}
\left\langle\beta_{i}, \beta_{j}\right\rangle & =N \sum_{\substack{a: \tau(a)=i-1 \\
b: \tau(b)=j-1}} \psi_{a}^{(\Phi)} \psi_{b}^{(\Phi)}\left\langle\alpha_{a}, \alpha_{b}\right\rangle \\
& =N \sum_{\substack{a: \tau(a)=i-1 \\
a}} \psi_{a}^{(\Phi)} \sum_{b}\left(G_{p}\right)_{a b} \psi_{b}^{(\Phi)} \\
& =N \sum_{\substack{a: \tau(a)=i-1 \\
\text { (\$) }}}\left(\psi_{a}^{(\Phi)}\right)^{2} \gamma_{p}^{(\Phi)} \\
& =\gamma_{p}^{(\Phi)}
\end{aligned}
$$

where $p=j-i \bmod N \neq 0 \bmod N$ and we have used again $\sum_{a: \tau(a)=i}\left|\psi_{a}^{(\Phi)}\right|^{2}=\frac{1}{N}$. The resulting folded graphs are like the starpolygons mentioned above in the discussion of $\widehat{s l}(N)_{1}$ (sect. 3.2), but with edges now carrying the numbers $\gamma_{p}^{(1)}$, i.e. $\gamma_{p}^{(0)}$ or $\gamma_{p}^{(\rho)}$ of eq. (12). In the case of $N=2$ class II graphs, (i.e. starting from an ordinary 
$A D E$ graph), one obtains the Coxeter graph $I_{2}(h)$. For class I graphs, the graph $G$ has two vertices with $G_{12}=2$ edges between them, this is -up to the change of sign convention already mentioned in footnote 3 the graph usually represented as $\iota^{\infty}$ and denoted in the literature $\hat{A}_{1}$ or $\tilde{A}_{1}$ or $A_{1}^{(1)}$ (we shall use the first notation). For $N>2$, the edges of the folded graphs encode scalar products which are (generically) larger than two, exposing the hyperbolic nature of the geometry of the root system.
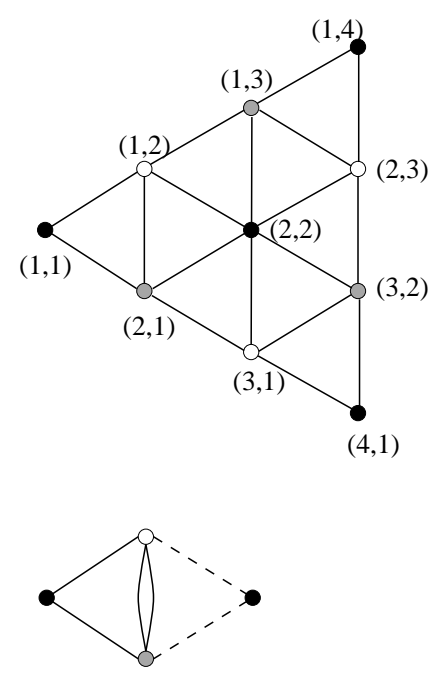
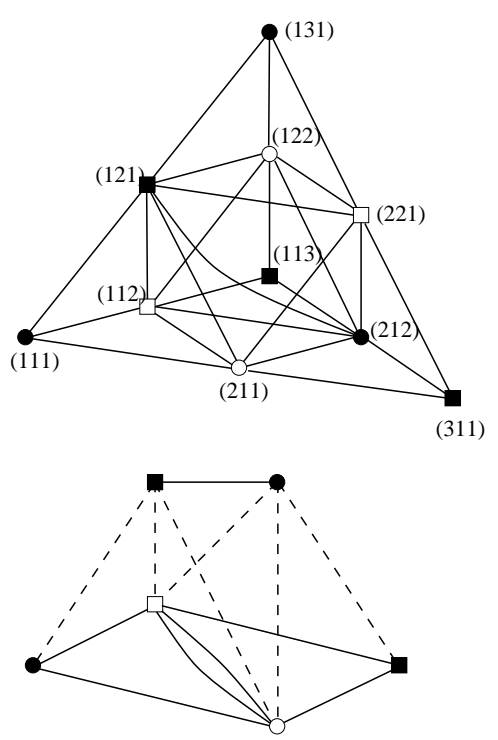

Fig. 4: The folding of $\mathcal{A}$ diagrams (top) into $\mathcal{B}$ diagrams (bottom). Left: $\widehat{s l}(3)$ at level 3; right: $\widehat{s l}(4)$ at level 2. Here for more clarity, we have replaced the notation $\frac{4}{4}$ (i.e. scalar products equal to $\sqrt{2}$ ) by a dashed line. In the $\mathcal{B}$ graphs: in the case of $\widehat{s l}(3)_{3}$, the left black dot represents the $\sigma$-orbit of $\lambda=(1,1)$, the right one $(2,2)$. In the case of $\widehat{s l}(4)_{2}$, the left black dot represents the $\sigma^{2}$-orbit of $(1,1,1)$, the upper one $(2,1,2)$. The right black square represents the orbit of $(3,1,1)$, the upper one $(1,2,1)$.

Another case of general validity is provided by the fusion graphs of $\widehat{s l}(N)_{k}$, when $k$ and $N$ are not coprimes $(N, k)=p$. Then it is easily seen that $\sigma^{p}$ is an automorphism of $\mathcal{P}_{++}^{(h=N+k)}$ which preserves the grading $\tau$. It is thus a $\mathbb{Z}_{N / p}$ automorphism in the sense of case 2) of Prop. 3.1 and may thus be used to fold the fusion graph. The result, which we denote $\mathcal{A}^{(h)} \mapsto \mathcal{B}^{(h)}$ by analogy with the conventional $A$ and $B$ Dynkin diagrams is illustrated in Fig. 4 in the two cases of $\widehat{s l}(3)_{3}$ and $\widehat{s l}(4)_{2}$. Note that this folding should not be confused with the orbifolding procedure $\mathcal{A}^{(h)} \mapsto$ $\mathcal{D}^{(h)}$ of $[\mathrm{Ko}, \mathrm{FeG}]$ (see Fig. 3); in the latter, the fixed points of $\sigma^{p}$ are taken with the multiplicity $N / p$ in the orbifold graph. These orbifold 
graphs may themselves be folded: see Fig.7 below.

Finally we notice that for a class I graph emanating from a finite subgroup $H$ of $S U(N)$ (see sect 1.3), a subalgebra of the $M$ algebra is nothing else than a subalgebra of the class algebra of $H$. Stability of Exp under $\sigma$ is equivalent to the stability of these classes under the $\mathbb{Z}_{N}$ center, and property $\wp$ follows from the positivity of the structure constants of the class and character algebras. Thus with any such subalgebra, we may associate a folding of the corresponding Dynkin diagram.

We now review the folding of the $N=2$ graphs, i.e. of the ordinary (simple or affine) Dynkin diagrams.

For class I, one may fold any simply laced affine Dynkin diagram onto $\hat{A}_{1}$ as just explained. In Fig. 5 this is displayed only for the folding of $\hat{A}_{2 n-1}$ into $\hat{A}_{1}$. All the other foldings exploit an obvious symmetry of one of the diagrams of type $\hat{A}, \hat{D}, \hat{E}_{6}$ or $\hat{E}_{7}$. One may fold $\hat{D}_{n+2}$ into $\hat{B}_{n+1}$ or into $\hat{C}_{n}$, and $\hat{D}_{2 p+2}$ into $\hat{B}_{p+1}$. Likewise, one may fold $\hat{E}_{7}$ into $\hat{F}_{4}$ or into $\hat{G}_{2}$. The latter may also be obtained from $\hat{E}_{6}$. Note that in order to discuss the folding of $\hat{A}_{2 n-1}$ into $\hat{C}_{n+1}$ or of $\hat{D}_{n+2}$ into $\hat{B}_{n+1}$, one cannot use the natural basis $\psi$ associated with characters of the corresponding subgroups of $S U(2)$, but one must choose another one in which positivity of the $M$ and $N$ algebras is lost but the existence of subalgebras is manifest. These cases thus escape the case 3) of Prop. 3.1 but are still covered by case 2 ).

For class II (fig 6), i.e. ordinary simple Dynkin diagrams, the situation is quite parallel. Any simply laced diagram with Coxeter number $h$ may be folded into $I_{2}(h)$. This is shown in Fig. 6 only for the $A_{n}$ graphs and for $D_{4} \mapsto I_{2}(6) \equiv G_{2}$. Some other foldings result from some obvious symmetry: $A_{2 k+1} \mapsto B_{k+1}, D_{2 p+2} \mapsto C_{2 p+1}, E_{6} \mapsto F_{4}$ (and also $D_{4} \mapsto G_{2}$ ). But the cases of $D_{6} \mapsto H_{3}$ or of $E_{8} \mapsto H_{4}$ require more ingenuity if one proceeds empirically. The virtue of the procedure discussed in the present paper is to yield these cases as well as the others as resulting from the discussion of sect. 3.2 and 3.3, namely from Prop. 3.1. In fact all cases but $D_{2 p+2} \mapsto C_{2 p+1}$ fall into case 3 ) of that Proposition. For the latter, as discussed before, to expose the $M$-subalgebra, one has to choose a basis in which positivity of the $M$ and $N$ is lost.

In these $N=2$ cases, where there is an a priori classification of all possible graphs with a positive definite or semi-definite metric $g[\mathrm{BH}]$, one may verify that the folding procedure based on $M$-subalgebras is exhaustive: it produces all non simply laced diagrams starting with the simply laced ones. It would be desirable to have such a result in general.

As for the folding of $N>2$ graphs, we content ourselves with a sample of graphs and foldings in Fig. 7. 

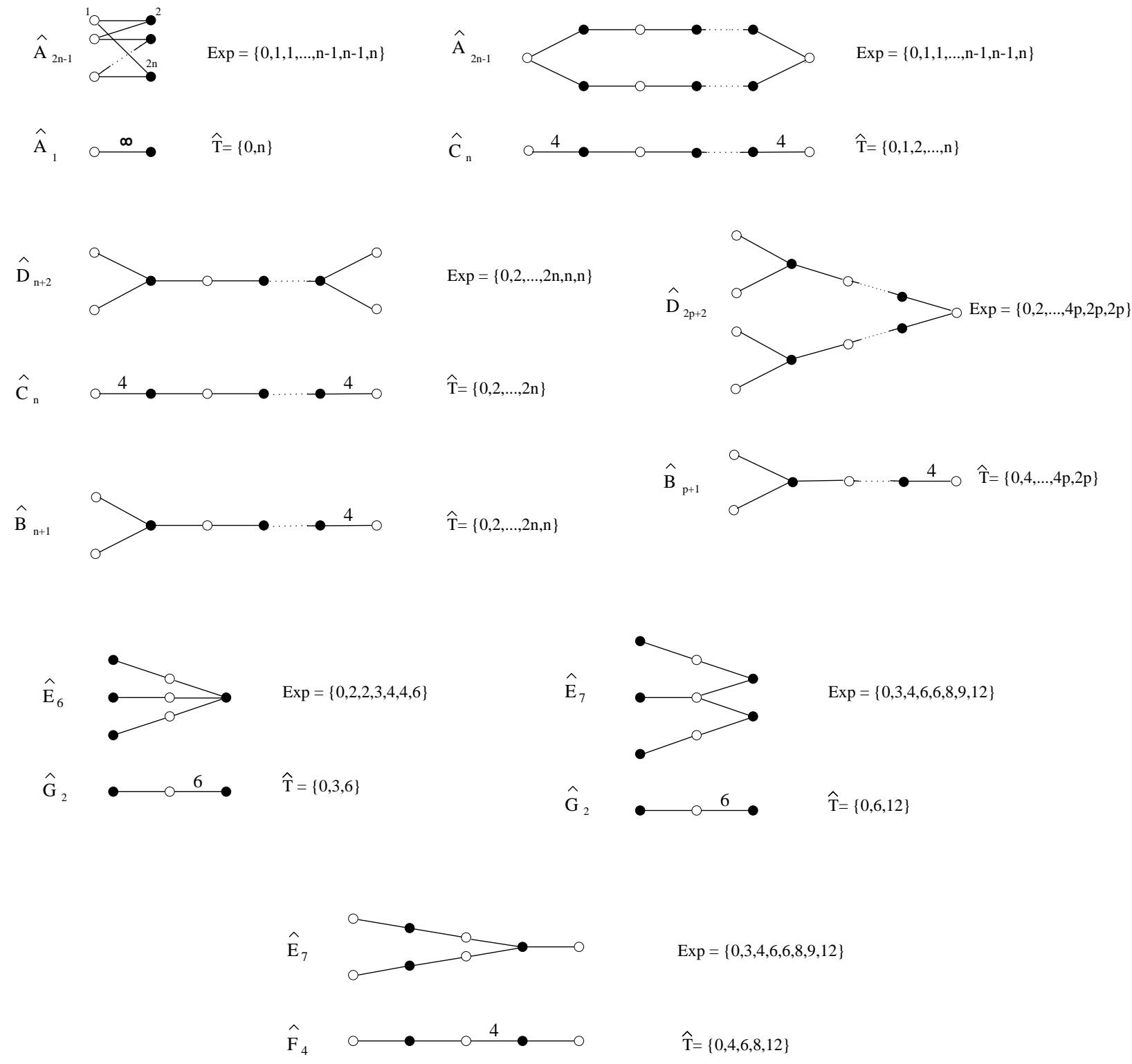

Fig. 5: The folding of $\hat{A}, \hat{D}, \hat{E}$ Dynkin diagrams. Classes $T_{i}$ encompass vertices on the same vertical. For the foldings $\hat{A}_{2 n-1}$ or $\hat{D}_{n+2}$ into $\hat{B}_{n+1}$ or $\hat{C}_{n}$, the parity of $n$ is irrelevant, i.e. the color of the rightmost vertices could be reversed.

\section{Relations with singularity theory}

It is well known that generalized Dynkin diagrams may be associated with singularities. In particular the $A D E$ diagrams are associated with simple singularities [AGZV]. A subclass of the diagrams studied in this paper may be given such an interpretation. 


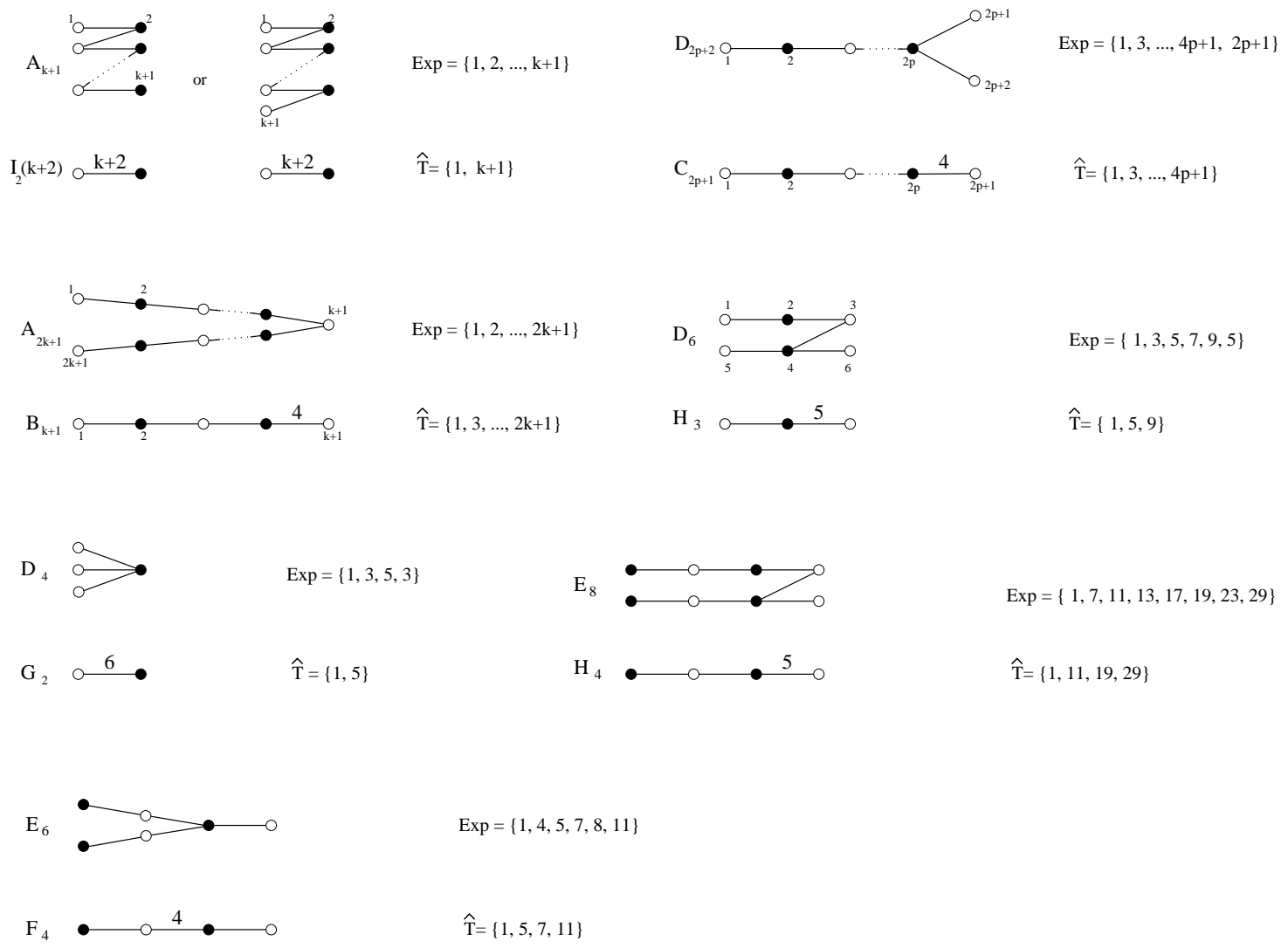

Fig. 6: The folding of $A D E$ Dynkin diagrams. Classes $T_{i}$ encompass vertices on the same vertical.

This should only be true for (some) graphs of class II. For a polynomial singularity $W=0$, one looks at the monodromy of homology cycles of a non-singular level set $\left\{x \in \mathbb{C}^{m},|x|<\delta \mid W(x)=\epsilon\right\}$ as $\epsilon$ circles around the origin [AGZV]. When $W$ is quasi-homogeneous, this monodromy operator is necessarily of finite order. If the putative Coxeter element $R$ discussed in sect. 2.2 is to be identified with this monodromy operator of the singularity, as is the case in many explicit cases, then graphs of class I for which $R$ has been proved to be of infinite order (Prop. 2.3) are excluded from this interpretation.

The case that is best understood is the class of "fusion graphs" of $\widehat{s l}(N)_{k}$. As explained in sect. 1.3, these graphs encode the fusion of integrable representations of $\widehat{s l}(N)_{k}$ by the fundamental representations. The fusion algebra of $\widehat{s l}(N)_{k}$ has been proved by Gepner [Ge] to be isomorphic to the quotient algebra $\mathbb{C}\left[X_{1}, \cdots, X_{N-1}\right] / \mathcal{J}\left(\partial_{i} \tilde{W}_{N, k}\right)$, quotient of the algebra of polynomials in $N-1$ variables by the ideal generated 
(a)

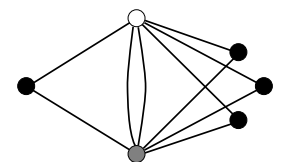

$\operatorname{Exp}=\{(1,1),(4,1),(1,4)$,thrice $(2,2)\}$

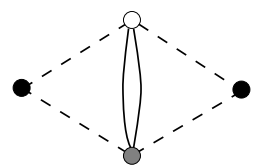

$\mathrm{T}=\{(1,1),(4,1),(1,4),(2,2)\}$

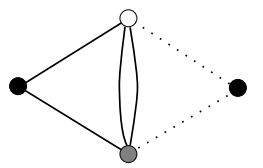

$\widehat{T}=\{(1,1),(4,1),(1,4),(2,2)\}$

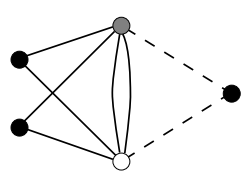

$\mathrm{T}=\{(1,1),(4,1),(1,4)$, twice $(2,2)\}$

(b)
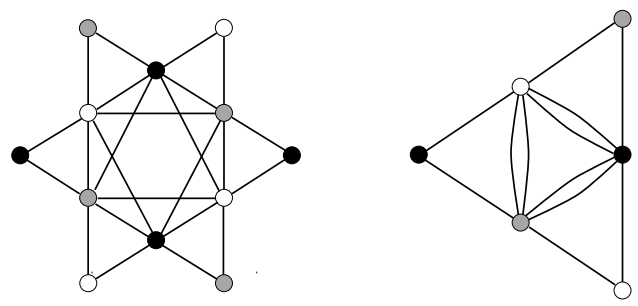

$\operatorname{Exp}=\{(1,1),(3,3),(4,1),(1,4)$ and their $\sigma-$ orbit

(c)
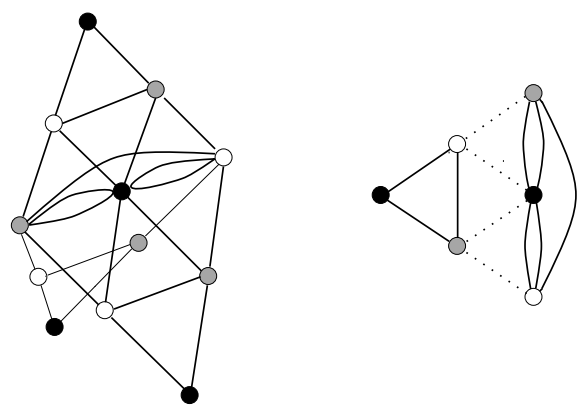

(d)

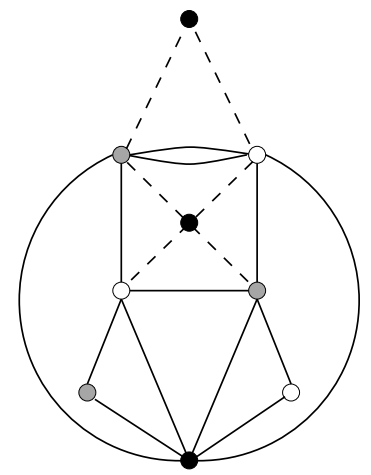

$\uparrow=\{(1,1),(3,3),(5,5)$ and their $\sigma$-orbit $\}$

$\operatorname{Exp}=\{(1,1),(10,1),(1,10),(5,5),(2,5),(5,2)$, twice $(3,3),(6,3),(3,6)\}$

Fig. 7: A sample of some $N=3$ diagrams and their folding. (a) the graph $\mathcal{D}^{(6)}$ of fig. 3 and three of its possible foldings; same convention as in fig. 4 for the dashed lines, the dotted lines stand for $\frac{6}{\text { (i.e. }}$ a scalar product equal to $\sqrt{3}$ ). (b) a graph of Coxeter number $h=8$, its set of exponents and a folding: the exponents of the subset $\hat{T}$ are underlined; (c) a graph of Coxeter number $h=12$, its set of exponents, a subset $\hat{T}$ underlined leads to the folding on the right; (d) folding of the graph of fig. $1(\mathrm{~b})$.

by the first partial derivatives of the "fusion potential"

$$
\tilde{W}_{N, k}\left(X_{1}, \cdots, X_{N-1}\right)=\left.\left(\frac{d}{d t}\right)^{k+N} \log \left(1-t X_{1}+\cdots+(-t)^{N-1} X_{N-1}+(-t)^{N}\right)\right|_{t=0}
$$

It is then very natural to consider the quasi-homogeneous part of this polynomial and to conjecture that the fusion graphs of the present paper are the Dynkin diagrams associated with the singularity

$$
W_{N, k}\left(X_{1}, \cdots, X_{N-1}\right)=\left.\left(\frac{d}{d t}\right)^{k+N} \log \left(1-t X_{1}+\cdots+(-t)^{N-1} X_{N-1}\right)\right|_{t=0} .
$$


This conjecture [Z] has now been proved by Gusein-Zade and Varchenko [GZV]. This proof has also the following interesting consequences:

- All the isomorphisms painfully discussed in a case-by-case analysis in [Z] are obtained in one shot! For example, the group associated with the graph $\mathcal{A}^{(5)}$ of fig.2 is isomorphic to the ordinary $D_{6}$ finite Coxeter group since both describe the singularity $W=X Y^{2}+X^{5}$. Likewise the $\mathcal{A}^{(6)}$ graph describes the same singularity $W=X^{6}+$ $a X^{2} Y^{2}+Y^{3}$ as the graph denoted $J_{10}$ in [AGZV].

- In all these isomorphisms of pairs of graphs/groups, the two root systems may be obtained from one another by a sequence of sign changes $\alpha_{a} \rightarrow-\alpha_{a}$ and braiding : $\left\{\alpha_{1}, \cdots, \alpha_{a-1}, \alpha_{a}, \cdots, \alpha_{n}\right\} \rightarrow$ $\left\{\alpha_{1}, \cdots, \alpha_{a}, S_{a} \alpha_{a-1}, \cdots, \alpha_{n}\right\}$.

- The monodromy operator of the singularity is the Coxeter element defined at the end of sect. 2.2 by the lexicographic ordering.

- Rank-level duality : the reflection groups associated with the fusion graphs of $\widehat{s l}(N+1)_{k}$ and $\widehat{s l}(k+1)_{N}$ are isomorphic. This too was conjectured in $[\mathrm{Z}]$ and proved in $[\mathrm{GZV}] .{ }^{4}$

In many cases, however, it is known that there is no description of the graph or group in terms of a single polynomial singularity. This is evidenced by looking at the characteristic polynomial of the monodromy operator. Suppose we have a singular polynomial $W\left(X_{1}, \cdots, X_{m}\right)$, quasihomogeneous of degree 1 with degree $\left(X_{\ell}\right)=d_{\ell}$, and suppose the eigenvalues of its monodromy operator are written as $(-1)^{m-1} \exp 2 i \pi \zeta_{j}$, then one proves $[\mathrm{Va}]$ that

$$
\mathcal{P}(t):=\sum_{j=1}^{n} t^{\zeta_{j}}=\prod_{\ell=1}^{m} \frac{t^{d_{\ell}}-t}{1-t^{d_{\ell}}}=t^{\Sigma d_{\ell}} \prod_{\ell=1}^{m} \frac{1-t^{1-d_{\ell}}}{1-t^{d_{\ell}}}
$$

The last product in (82) is the Poincaré polynomial (in some fractional power of $t$ ) counting the elements in the local ring of the singularity. For the Coxeter element $R$ of sect. 2.2, we may compute $\mathcal{P}(t)$ knowing the $\zeta_{\lambda}=-\frac{\left(e_{N}, \lambda\right)}{h}$. If $R$ is to be interpreted as the monodromy operator of a quasi-homogeneous singularity, then according to $(82), \mathcal{P}(t)$ must have its zeros on the circle $|t|=1$. Now, it is easy to check that in several cases discussed in the present paper, the operator $R$ does not meet this condition. An example is provided by the graph $\mathcal{D}^{(6)}$ of fig. 3 $\left(\right.$ alias $D_{4}^{(1,1)}$ of $\left.[\mathrm{S}]\right)$ for which

$$
\mathcal{P}(t)=(1+4 \sqrt{t}+t)
$$

4 Beware! this is not what is usually called the rank-level duality, which rather connects $s l(N)_{k}$ and $s l(k)_{N}$. There is, however, a relation between the two concepts $[\mathrm{NS}]$. 
the roots of which lie clearly out of the unit circle.

Finally we note that whenever a graph may be associated with a singularity, its various foldings are associated with boundary singularities, as discussed in [Y,Sh,AGZV].

All these connections with singularity theory have a natural transcription in "physical" language, in the context of two-dimensional $\mathcal{N}=2$ superconformal field theories and/or topological field theories. We shall only sketch the line of arguments. It is believed that there exist $\mathcal{N}=2$ superconformal and topological field theories associated with each of the graphs discussed here. Cases associated with a singularity are said to have a Landau-Ginsburg (L-G) potential. Not all theories possess such a L-G potential. In all cases, however, as already mentioned above, Cecotti and Vafa [CV] have found that in the study of the so-called $t t^{*}$ equations, one encounters a monodromy operator with very specific properties. It is suggested that the Coxeter element of this paper is the monodromy operator of Cecotti and Vafa. On the other hand, Dubrovin has developped a geometric interpretation of the axioms of two-dimensional topological field theories (tft), in terms of Frobenius manifolds. There too, a differential system which relates the flat coordinates of two independent flat metrics plays a central role, and its monodromy group is -at least in some cases- generated by reflections. There are many indications that the reflection group of the present paper describe this monodromy group (or a subgroup of it) in the case of topological theories based on $\operatorname{sl}(N)$. The challenge would be to reconstruct the topological field theory, at least in genus zero a solution of the Witten-Dijkgraaf-Verlinde-Verlinde equations-, from the graph. This presumably involves the study of the space of orbits of the group, in the spirit of [D]. In that context, going from a graph to a folded graph must produce another topological theory, obtained by restricting the moduli space of the original one. This is what happens in the simplest cases of the minimal tft's [D,Zu].

\section{Conclusion and perspectives}

The present paper has considered a class of graphs which generalize in a natural way the ordinary Dynkin diagrams and thus lead to reflection groups. These graphs satisfy a certain number of axioms. Admittedly, these axioms lack elegance and conciseness! A more compact and conceptually simpler way of encapsulating them would be welcome.

We have shown that many solutions may be obtained in a systematic way by folding. Folding of graphs is usually found using their symmetries. Roughly speaking, these symmetries of the vertex set imply 
restrictions in the dual ("Fourier") exponent set, consistent with their algebra ( $M$ algebra). We have found that conversely and more generally, such restrictions -with additional technical assumptions- may lead to new foldings not associated with an obvious symmetry.

As already pointed out, our analysis in terms of $C$-algebras and subalgebras relies on explicit choices of basis in these algebras (i.e. explicit choices of the eigenvectors $\psi$ ). It would be desirable to have a more intrinsic and geometric standpoint. The same applies to property $\wp$, which appears as a useful technical assumption, whose intrinsic meaning remains however obscure.

Another direction which deserves more work is the connection with singularity theory. In particular, in cases not associated with a quasihomogeneous polynomial, is there a substitute?

Finally the connections with superconformal field theories and topological field theories reviewed at the end of previous section are still awaiting a thorough discussion.

\section{Acknowledgements}

I want to thank the Taniguchi Foundation and the organisors of this Symposium, K. Saito, M. Kashiwara, A. Matsuo and I. Satake for providing exceptional conditions of work and of scientific exchanges in a beautiful location. I benefited a lot from discussions with many participants, especially K. Saito, I. Satake and P. Slodowy. I would like also to acknowledge the stimulating criticisms and suggestions of M. Bauer, P. Di Francesco, V. Petkova and A. Varchenko during the preparation of this paper.

\section{References}

[AGZV] V.I. Arnold, and S.M. Gusein-Zade, A.N. Varchenko, Singularities of differentiable maps, Birkäuser, Basel 1985.

[BH] N. Bourbaki, Groupes et Algèbres de Lie, chap. 4-6, Masson 1981; J.E. Humphreys, Reflection Groups and Coxeter Groups, Cambridge Univ. Pr. 1990.

[BI] E. Bannai, T. Ito, Algebraic Combinatorics I: Association Schemes, Benjamin/Cummings (1984).

[BLM] S. Berman, Y.S. Lee and R.V. Moody, J. Algebra 121 (1989) 339-357.

[C] H.S.M. Coxeter, Ann. Math. 35 (1934) 588-621.

[CV] S. Cecotti and C. Vafa, Nucl.Phys.367 (1991) 359-461; Comm. Math. Phys.158 (1993) 569-644 .

[D] B. Dubrovin, Nucl. Phys. B 379 (1992) 627-689: hep- 
th/9303152; Springer Lect. Notes in Math. 1620 (1996) 120348: hep-th/9407018; B. Dubrovin and Y. Zhang, Extended affine Wey groups and Frobenius manifolds, hep-th/9611200.

[DFZ1] P. Di Francesco and J.-B. Zuber, Nucl. Phys. B338 (1990) 602-646.

[DFZ2] P. Di Frahcesco and J.-B. Zuber, in Recent Developments in Confornal Field Theories, Trieste Conference, 1989, S. Randjbar-Daemi, E. Sezgin and J.-B. Zuber eds., World Scientific 1990; P. Di Francesco, Int.J.Mod.Phys. A7 (1992) 407-500.

[FFK] W.M. Fairpairn, T. Fulton and W.H. Klink, J. Math. Phys 5 (1964) 103\$-1051.

[FG] P. Fendley and P. Ginsparg, Nucl. Phys. B324 (1989) 549-580.

[FH] W. Fulton and J. Harris, Representation Theory, Springer Verlag, ex 2.3才 page 25, corr P 517.

[G] D. Gepner, Comm. Math. Phys. 141 (1991) 381-411.

[GHJ] F. Goodman, P. de la Harpe and V.F.R. Jones, Coxeter graphs and towers of algebras, 14 MSRI Publ., Springer (1989).

[GZV] S.M. Gusein-Zade and A. Varchenko, Verlinde algebras and the intersection form on vanishing cycles, hep-th/9610058.

[K] B. Kostant Société Mathématique de France, Astérisque (1988) 209-255.

[Ko] I.K. Kostoy, Nucl. Phys. B 300 [FS22] (1988) 559-587.

[KP] V.G. Kac and D.H. Petersen Adv. Math. 53 (1984) 125-263.

[MK] J. MacKay Proc. Symp. Pure Math. 37 (1980 183-186.

[MP] R.V. Moody and J. Patera, J.Phys.A 26 (1993) 2829-2853.

[NS] S.G. Naculich and H.J. Schnitzer, Superconformal coset equivalence from level-rank duality, hep-th/9705149.

[O] A. Ocneanu, communication at the Workshop Low Dimensional Topology, Statistical Mechanics and Quantum Field Theory, Fields Inst tute, Waterloo, Ontario, April 26-30, 1995.

[P] V. Pasquie:, J.Phys. A20 5707-5717 (1987); Thèse d'Etat, Orsay, 1988.

[Pe] V.B. Petkova, private communication and to appear.

[PZ] V.B. Petkqva and J.-B. Zuber, Nucl. Phys. B B463 (1996) 161-193: h\&p-th/9510175; Conformal Field Theory and Graphs, hep-th/9701103.

[S] K. Saito, Publ. RIMS, Kyoto Univ. 21 (1985) 75-179, 26 (1990) 15-78; K. Saito and T. Takebayashi, preprint RIMS-1089.

[Sh] O.P. Shcherbak, Russ. Math. Surveys 43:3 (1988) 149-194.

[St] J. Steenbripk, Compositio Math. 34 (1977) 211-223.

[Va] A.N. Varchenko, Inv. Math. 37 (1976) 253-262.

[Ve] E. Verlinde, Nucl. Phys. B300 [FS22] (1988) 360-376.

[Y] J. Sekiguch i and T. Yano, Sci. Rep. Saitama Univ. IX (1980) 33-44; T. Yano, ibid. 61-70. 
[Z] J.-B. Zuber, Comm. Math. Phys. 179 (1996) 265-294.

[Zi] J.-B. Zuber, Conformal, Integrable and Topological Theories, Graphs and Coxeter Groups, in XIth International Congress of Mathematical Physics, Paris July 1994, D. Iagolnitzer edr, International Press 1995, p 674-689, hep-th/9412202.

[Zr] J.-B. Zuber, C-algebras and their applications to reflection groups and conformal field theories, proceedings of the RIMS Symposium, Kyoto, 16-19 December 1996, hep-th/9707034.

[Zu] J.-B. Zuber, Mod. Phys. Lett A8 (1994) 749-760.

Jean-Bernard Zuber

Service de Physique Théorique,

CEA Saclay, F-91191 Gif sur Yvette Cedex, (France)

email: zuber@spht.saclay.cea.fr 